\title{
The Hippo signaling pathway is required for salivary gland development and its dysregulation is associated with Sjogren's syndrome
}

Tone B Enger ${ }^{1}$, Arman Samad-Zadeh², Meghan P Bouchie², Kathrine Skarstein ${ }^{3}$, Hilde K Galtung ${ }^{4}$, Toshiyuki Mera ${ }^{5}$, Janice Walker ${ }^{6}$, A Sue Menko ${ }^{6}$, Xaralabos Varelas ${ }^{7}$, Denise L Faustman ${ }^{5}$, Janicke L Jensen ${ }^{1}$ and Maria A Kukuruzinska²

Sjogren's syndrome (SS) is a complex autoimmune disease that primarily affects salivary and lacrimal glands and is associated with high morbidity. Although the prevailing dogma is that immune system pathology drives SS, increasing evidence points to structural defects, including defective E-cadherin adhesion, to be involved in its etiology. We have shown that E-cadherin has pivotal roles in the development of the mouse salivary submandibular gland (SMG) by organizing apical-basal polarity in acinar and ductal progenitors and by signaling survival for differentiating duct cells. Recently, E-cadherin junctions have been shown to interact with effectors of the Hippo signaling pathway, a core pathway regulating the organ size, cell proliferation, and differentiation. We now show that Hippo signaling is required for SMG-branching morphogenesis and is involved in the pathophysiology of SS. During SMG development, a Hippo pathway effector, TAZ, becomes increasingly phosphorylated and associated with E-cadherin and $\alpha$-catenin, consistent with the activation of Hippo signaling. Inhibition of Lats2, an upstream kinase that promotes TAZ phosphorylation, results in dysmorphogenesis of the SMG and impaired duct formation. SMGs from non-obese diabetic mice, a mouse model for SS, phenocopy the Lats2-inhibited SMGs and exhibit a reduction in E-cadherin junctional components, including TAZ. Importantly, labial specimens from human SS patients display mislocalization of TAZ from junctional regions to the nucleus, coincident with accumulation of extracellular matrix components, fibronectin and connective tissue growth factor, known downstream targets of TAZ. Our studies show that Hippo signaling has a crucial role in SMG-branching morphogenesis and provide evidence that defects in this pathway are associated with SS in humans.

Laboratory Investigation (2013) 93, 1203-1218; doi:10.1038/labinvest.2013.114; published online 30 September 2013

KEYWORDS: E-cadherin adhesion; Hippo signaling; salivary gland; Sjogren's syndrome; TAZ

Sjogren's syndrome (SS) is a disabling autoimmune disease of the salivary and lacrimal glands whose manifestations include hyposalivation and ocular dryness. ${ }^{1}$ The central dogma in SS and autoimmunity, in general, has been that the T-cell receptor determines target specificity and that immune system pathology is the sole explanation for disease. However, this dogma is increasingly challenged by the fact that in diabetes and SS-associated neuropathology, structural defects often exist before the onset of autoimmune disease. ${ }^{2-6}$ Further, recent findings in non-obese diabetic (NOD) mice, a mouse model for SS, suggest a direct role for the tissue itself in disease targeting. ${ }^{7,8}$ As little is known about the etiology of SS, identification of the molecular events that are essential for development of the salivary gland and whose dysregulation gives rise to structural defects in NOD mice that mimic SS in humans is critical.

Development of the salivary gland has been most extensively studied in the mouse salivary submandibular gland (SMG) as a model system. The SMG belongs to a group of epithelial tissues that develop through branching

\footnotetext{
${ }^{1}$ Department of Oral Surgery and Oral Medicine, University of Oslo, Oslo, Norway; ${ }^{2}$ Department of Molecular and Cell Biology, Boston University School of Dental Medicine, Boston, MA, USA; ${ }^{3}$ Section for Pathology, The Gade Institute, University of Bergen, Haukeland University Hospital, Bergen, Norway; ${ }^{4}$ Department of Oral Biology, University of Oslo, Oslo, Norway; ${ }^{5}$ Immunobiology Laboratories, Harvard Medical School and Massachusetts General Hospital, Boston, MA, USA; ${ }^{6}$ Department of Pathology, Anatomy and Cell Biology, Thomas Jefferson University, Philadelphia, PA, USA and ${ }^{7}$ Department of Biochemistry, Boston University School of Medicine, Boston, MA, USA

Correspondence: Dr MA Kukuruzinska, PhD, Department of Molecular and Cell Biology, Boston University Medical Campus, 72 E Concord Street, E428, Boston, MA 02118, USA.
}

E-mail: mkukuruz@bu.edu

Received 11 July 2013; revised 30 August 2013; accepted 30 August 2013 
morphogenesis, a process regulated by the reciprocal cross talk between the epithelium and mesenchyme, as well as by interactions with the endothelium and the neuronal system. ${ }^{9-16}$ The SMG has become a useful tool for the identification of molecular events regulating this developmental process, in part, because of the ability to grow early embryonic SMG organ cultures, which provides an excellent ex vivo model that mimics branching morphogenesis in vivo. ${ }^{12,15}$ Using this system, we have identified E-cadherin, the primary epithelial cell-cell adhesion receptor that organizes adherens junctions (AJs), as a principal regulator of morphogenetic changes during SMG development. E-cadherin regulates branching morphogenesis by establishing apical-basal polarity in acinar and ductal progenitors and by protecting differentiating duct cells within newly forming ductal lumens from apoptotic death. ${ }^{16}$ However, cues that lie downstream of E-cadherin in SMG development are poorly understood.

Recently, E-cadherin-mediated cell adhesion has been shown to influence the activity of the Hippo pathway, a conserved signaling pathway critical for regulating cell fate, tissue homeostasis, and organ growth. ${ }^{17-20}$ In mammals, the primary downstream effectors of the Hippo pathway are the paralogous proteins TAZ and YAP. The functions of TAZ and YAP are determined by their nuclear-cytoplasmic localization, which is regulated by phosphorylation. The Lats1 and Lats2 kinases are the primary kinases described to regulate TAZ and YAP localization by promoting their phosphorylation on residues that induce binding to 14-3-3, a family of conserved regulatory proteins that interact with cell polarity and adhesion proteins to sequester TAZ and YAP in the cytoplasm. Lats kinase inhibition promotes TAZ and YAP nuclear accumulation, subsequently inducing TAZ and YAP activity as transcriptional regulators. ${ }^{18,21}$

Salivary glands and pancreatic islets from NOD tissues uniformly display lack of E-cadherin adhesion in situ and altered cellular adhesion in isolated cells. ${ }^{8}$ As Hippo signaling is regulated by $\mathrm{E}$-cadherin-mediated adhesion through binding to $\alpha$-catenin and tight junction-associated Crumbs polarity complexes, ${ }^{22-25}$ we hypothesized that defects in this pathway promote NOD dysfunction and SS phenotype in humans. In this study, we examine the Hippo pathway in mouse SMG embryonic development and show that it has a role in E-cadherin-mediated apical-basal polarity in differentiating acinar and ductal progenitors and that it has critical roles in secondary duct formation. We also provide evidence that defective Hippo signaling contributes to the structural defects in the NOD SMG, and, importantly, deregulated Hippo signaling is a feature of human SS.

\section{MATERIALS AND METHODS Mouse Strains}

For mouse studies, CD1 wild-type and NOD mouse strains were used. The day the vaginal plug was detected was considered embryonic day 0.5 (E 0.5). All animal experiments were approved by the Institutional Animal Care and Use Committee at the Boston University Medical Center.

\section{Minor Human Salivary Glands}

Formalin-fixed, paraffin-embedded lower lip minor salivary glands were collected with a written consensus from three different biobanks in Oslo, Norway. The Norwegian Committee of Ethics approved the use of the biopsies in the study. To assure proper diagnosis, an oral pathologist re-evaluated all biopsies blindly using the American-European Consensus Criteria (AECC) to determine the focus score (the number of focal mononuclear cell infiltrates with $\geq 50$ mononuclear cells per $4 \mathrm{~mm}^{2}$ ). Minor salivary glands evaluated for SS, but not fulfilling AECC criteria, served as controls. Patients with SSA or SSB autoantibodies, or patients diagnosed with secondary SS, were excluded as controls. For each marker analyzed by immunostaining, a minimum of six biopsies from SS compatible patients and six non-compatible controls were used.

\section{SMG Organ Cultures}

SMG salivary gland rudiments were dissected from embryos at E13.5-E18.5, cultured on Whatman Nucleopore Tracketch filters (GE healthcare, Buckinghamshire, UK) and grown in an air/medium interface at $37^{\circ} \mathrm{C}$ in a humidified $5 \% \mathrm{CO}_{2}$ atmosphere. ${ }^{16}$ The filters were placed on top of $200 \mu \mathrm{l}$ media containing DMEM/F12 (ATCC, VA, USA) supplemented with $150 \mu \mathrm{g} / \mathrm{ml}$ vitamin C, $50 \mu \mathrm{g} / \mathrm{ml}$ transferrin, $100 \mu \mathrm{g} / \mathrm{ml}$ penicillin, and $100 \mu \mathrm{g}$ streptomycin in $30 \mathrm{~mm}$ glass bottom microwell dishes (MatTek, MA, USA) with six glands per filter. During transfection with small interfering RNA (siRNA), penicillin and streptomycin were omitted from the growth media. For functional studies using Lats2 siRNA, glands were photographed at 0,24 , and $48 \mathrm{~h}$ using a Nikon Eclipse TS100 microscope (Nikon, Tokyo, Japan). For analyses of SMGs treated with Lats2 siRNA, 23 silenced and 23 non-silenced glands were analyzed to measure the gland area and number of buds using ImageJ software (version 1.45s).

\section{Antibodies}

Antibodies to TAZ, E-cadherin, fibronectin, IQGAP1, and $\beta$-catenin were purchased from BD Transduction Laboratories, CA, USA. Two antibodies to $\alpha$-catenin, one produced in mouse and one produced in rabbit, were obtained from Sigma, MO, USA and BD Transduction Laboratories, respectively. The antibody to phospho-YAP, also recognizing phosho-TAZ, was obtained from Cell Signaling Technology, MA, USA. The antibody to YAP, also recognizing TAZ, was purchased from Santa Cruz Biotechnology, CA, USA. Although these antibodies recognize both TAZ and YAP, the differences in the molecular weights of TAZ $(55 \mathrm{kDa})$ and YAP $(65 \mathrm{kDa})$ make them easily distinguishable. Antibodies to connective tissue growth factor (CTGF), Ki67, and total YAP were purchased from Abcam, MA, USA. 


\section{Immunofluorescence Analyses}

Formalin-fixed, paraffin-embedded human labial salivary gland biopsies were cut into $4-6 \mu \mathrm{m}$ sections using a Leica serial Microtome (Leica Instruments, Solms, Germany) and placed on SuperFrost Plus microscope slides (Thermo Scientific, MA, USA). Before staining, each slide was rinsed in distilled water before heat-induced epitope retrieval in a microwave using 9:1 Retrievit-6 Target Retrieval Solution (Biogenex, CA, USA) and MilliQ water. Slices were cooled for 20 min before overnight blocking in 10\% goat serum (Sigma) at $4{ }^{\circ} \mathrm{C}$. Sections were incubated in primary antibodies diluted in PBS-T $(20 \mathrm{mM}$ Tris, $137 \mathrm{mM} \mathrm{NaCl}, 0.1 \%$ Tween $20, \mathrm{pH}$ 7.6) with $10 \% \mathrm{BSA}$ for $1 \mathrm{~h}$ at room temperature (RT). The slides were washed in PBS-T before incubating in goat anti-mouse/rabbit AlexaFlour 594 (Invitrogen, NY, USA) or goat anti-mouse/rabbit FITC-tagged (Invitrogen) secondary antibodies diluted at 1:100 in PBS-T, and counterstained for nuclei with 4,6-diamidino-2-phenylindole (DAPI; Invitrogen) at RT for $30 \mathrm{~min}$. The slides were then washed three times in PBS-T before mounting using Vectashield (Vector Laboratories, CA, USA) and microscope cover glass (Fisher Scientific, MA, USA). For negative controls, the primary antibodies were omitted.

For whole-mount immunofluorescence of mouse SMGs, E13.5, E15.5, and E18.5 glands were grown on filters for $2 \mathrm{~h}$ before fixation in $3.7 \%$ paraformaldehyde for $40 \mathrm{~min}$ at RT. The glands were then washed in PBS and permeabilized using $0.1 \%$ Triton-X in PBS for $20 \mathrm{~min}$ (E13.5) or $40 \mathrm{~min}$ (E15.5 and E18.5) at RT. After permeabilization, the glands were washed three times in PBS-T before overnight blocking in $10 \%$ goat serum, $10 \%$ BSA, and PBS-T at $4{ }^{\circ} \mathrm{C}$. Primary antibodies were diluted 1:100 in 10\% BSA and PBS-T and incubated for $3.5 \mathrm{~h}$ at RT. The glands were washed in PBS-T before adding FITC-conjugated secondary antibodies (Invitrogen) at 1:100 dilution in PBS-T for $1.5 \mathrm{~h}$ at RT. The secondary antibodies were removed, and Rhodamine Phalloidin (Invitrogen) at 1:100 and TO-PRO-3 iodide (Invitrogen) at 1:1000 were counterstained for F-actin and nuclei, respectively, for an additional $30 \mathrm{~min}$ at RT. The glands were then washed overnight and mounted on SuperFrost Plus microscope slides (Thermo Scientific) using $15 \mu \mathrm{l}$ Vectashield (Vector Laboratories). The immunostained glands were then analyzed using a Zeiss LSM 510 confocal microscope as previously described. ${ }^{16}$

\section{Tissue Extraction}

SMGs were immediately frozen on dry ice following dissection and were kept at $-80^{\circ} \mathrm{C}$ before protein extraction. For preparation of total tissue lysates, SMGs were extracted in OGT buffer $(10 \mathrm{mM}$ imidazole, $3 \mathrm{mM}$ sodium pyrophosphate, $100 \mathrm{mM} \mathrm{NaCl}, 1 \%$ Triton-X, $5 \mathrm{mM}$ EDTA, $1 \mathrm{mM}$ $\mathrm{MgCl}_{2}, 45 \mathrm{mM} n$-Octyl $\beta$-D glucopyranoside) supplemented with a protease inhibitor cocktail (Sigma) and $100 \mathrm{mM}$ PMSF. Whole-tissue lysates were used for detection of selected proteins and protein complexes by immunoblot and immunoprecipitation. Protein concentration was determined using the BCA assay (Thermo Scientific).

\section{Immunoblot and Co-Immunoprecipitation}

For biochemical analyses, 10-15 glands from E13.5, 6-8 glands from E15.5, or 2-4 glands from E18.5 were collected from the same litter and kept on dry ice before protein extraction as described above. For each embryonic time point, $50 \mu \mathrm{g}$ of protein was used with reducing $2 \times$ sample buffer (Sigma). The samples were heated at $95^{\circ} \mathrm{C}$ for $5 \mathrm{~min}$ before being fractionated on 7.5\% SDS-PAGE. The gel was electrophoretically transferred onto PVDF membranes (Invitrogen) at $60 \mathrm{~V}$. The membrane was blocked for $1 \mathrm{~h}$ in $10 \%$ milk before overnight incubation in primary antibody at $4{ }^{\circ} \mathrm{C}$, followed by $1 \mathrm{~h}$ incubation at $27^{\circ} \mathrm{C}$ using secondary horseradish peroxidase-conjugated antibodies (Sigma). ECL plus detection reagent (GE Healthcare) was used for visualization of bands, and quantification analysis of band intensity was performed using ImageJ software (version 1.45s).

For analyses of protein interactions by co-immunoprecipitation (co-IP), $300 \mu \mathrm{g}$ of total tissue lysate was prepared from either E15.5 or E18.5 glands. Unspecific binding to $\operatorname{IgG}$ was removed by preclearing with normal mouse IgG (Santa Cruz Biotechnology) for $1 \mathrm{~h}$. Samples were incubated with primary antibody for $2 \mathrm{~h}$ followed by incubation with protein agarose beads (GE Healthcare) for $1 \mathrm{~h}$ as described previously. ${ }^{16,26}$ Immunoprecipitates were subjected to electrophoresis under either reducing or non-reducing conditions, as described above.

\section{RNA Interference}

siRNAs targeting Lats2 were obtained as SMART-pool sequences (Dharmacon, CO, USA), whereas the non-silencing negative control siRNA was from Qiagen. To determine optimal siRNA concentrations, different amounts ranging from 100 to $400 \mathrm{nM}$ were tested for various time periods. The most distinct phenotype was observed using $400 \mathrm{nM}$ siRNA at $48 \mathrm{~h}$. From each mouse embryo, one gland was used for silencing, whereas the corresponding gland was used as a non-silencing control. Six glands were placed on each filter and transfected using RNAiFect (Qiagen) with either $400 \mathrm{nM}$ of siRNA targeting Lats2 or a non-silencing, scrambled control. BLOCK-iT Fluorescein Oligo (Invitrogen) was co-transfected with Lats2 siRNA to mark transfection efficiency. After transfection, glands were incubated at $37^{\circ} \mathrm{C}$ for $48 \mathrm{~h}$ and frozen in sterile tubes for protein or RNA extraction or fixed in $3.7 \%$ paraformaldehyde before immunostaining.

\section{RNA Extraction and RT-PCR}

RNA from SMGs was extracted using the Qiagen RNeasy Minikit, and cDNA was prepared using SuperScript III (Invitrogen). Gene expression profiles were generated by normalizing the threshold cycle number of Lats2 with housekeeping gene $18 \mathrm{~S}$ and by comparing the gene 
a
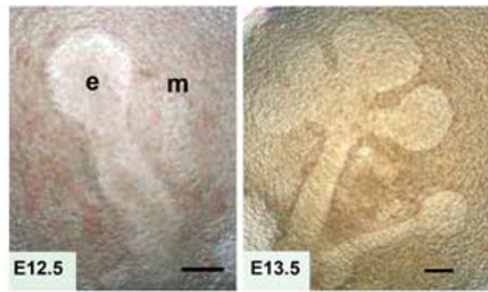

b
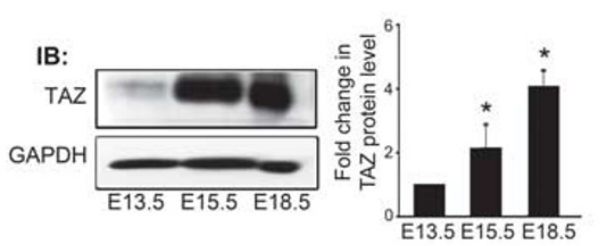

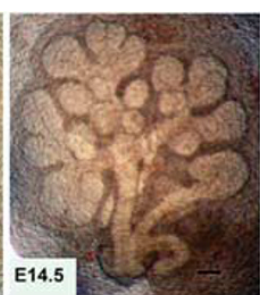

C

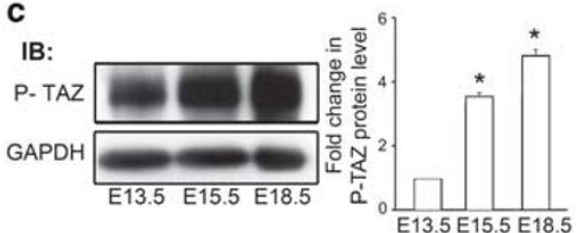

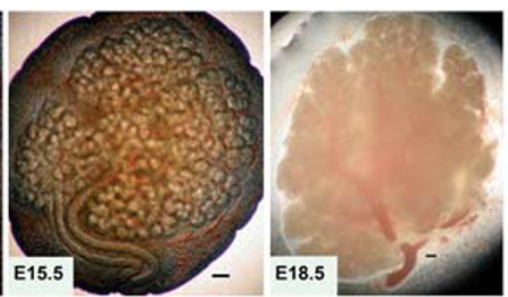

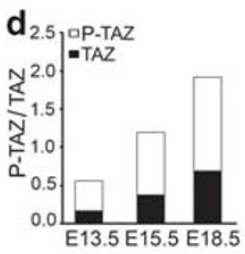

e

TAZ

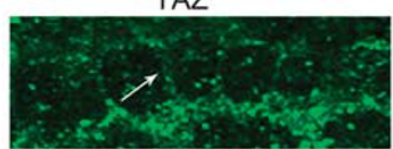

F-actin
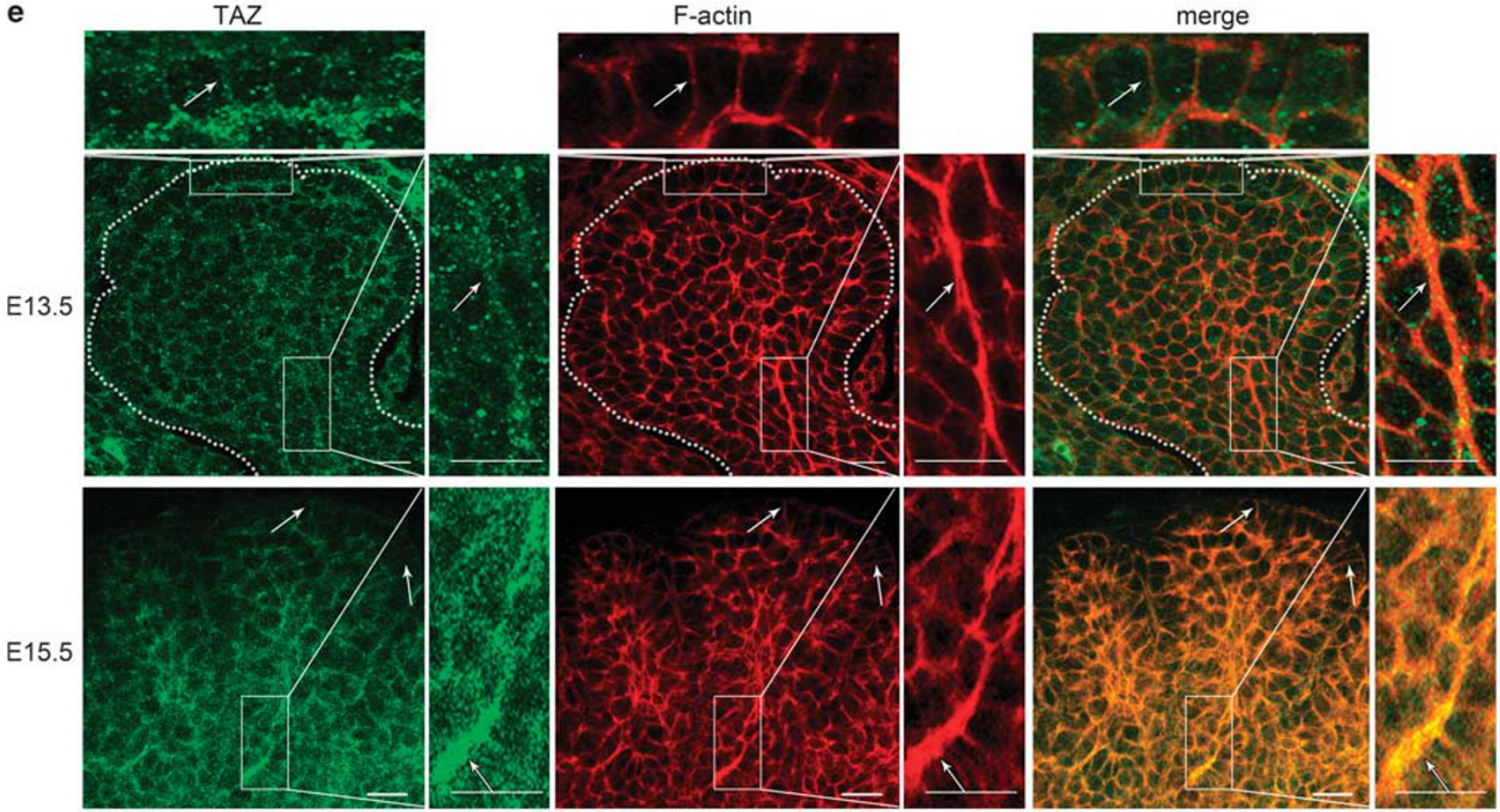

E15.5
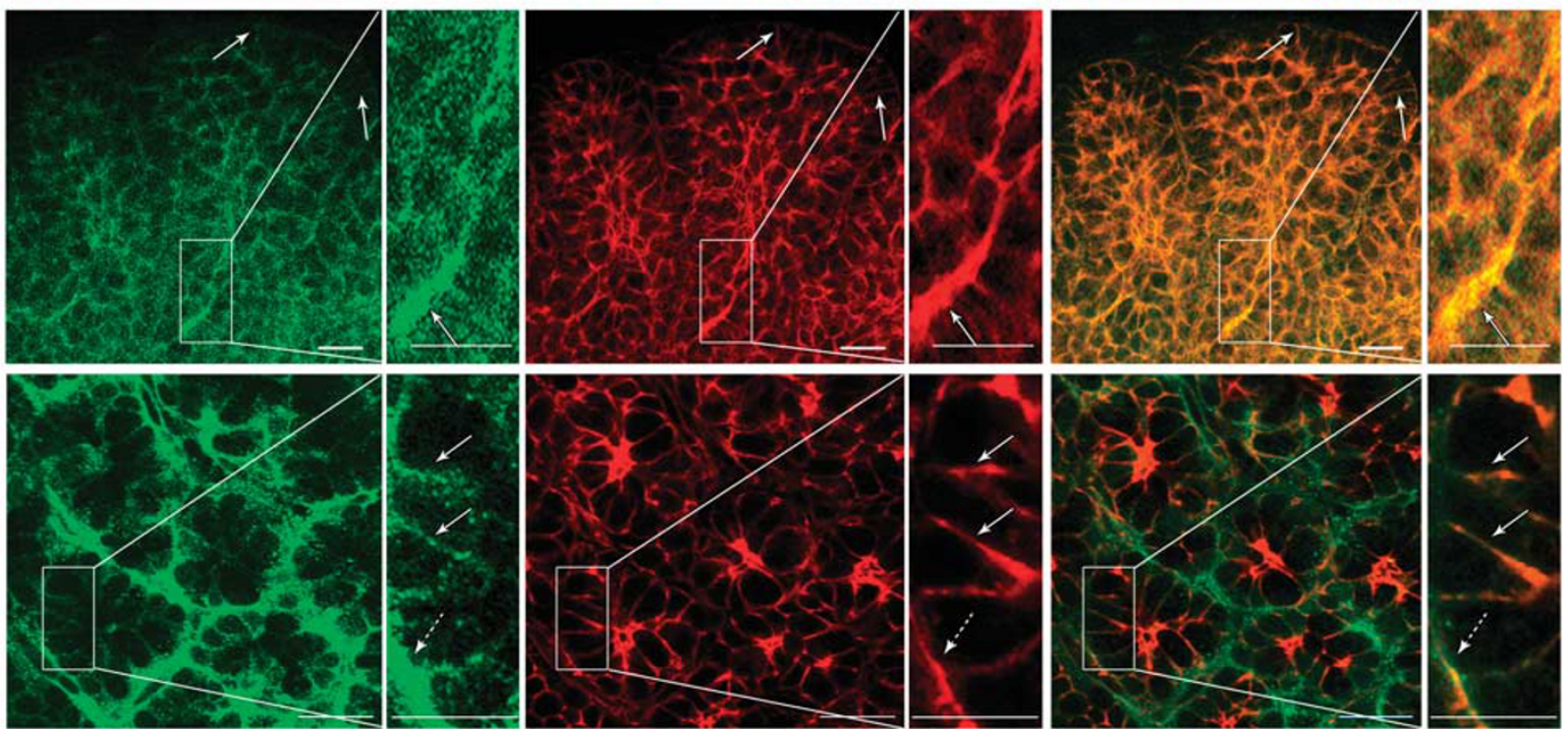

E18.5

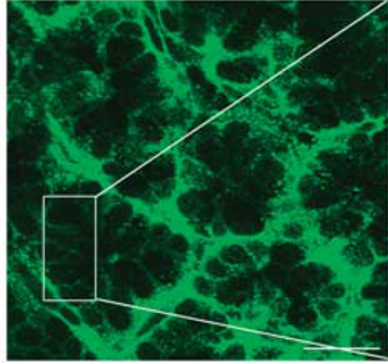

Figure 1 Expression and localization of TAZ during salivary submandibular gland (SMG) embryonic development. (a) Phase microscopy images of CD1 mice epithelial buds (e) and surrounding mesenchyme $(\mathrm{m})$ isolated from embryonic days E12.5-E18.5 during SMG-branching morphogenesis. Size bar: $20 \mu \mathrm{m}$. (b) Immunoblot of TAZ SMG expression levels at E13.5, E15.5, and E18.5. Bar graph, fold change in TAZ levels after normalization to glyceraldehyde 3-phosphate dehydrogenase (GAPDH), ${ }^{*} P<0.05$. (c) Immunoblot of $\mathrm{p}$-TAZ expression levels at E13.5, E15.5, and E18.5. Bar graph, fold change in $p$-TAZ levels after normalization to GAPDH, ${ }^{*} P<0.05$. (d) Ratio of $\mathrm{p}-\mathrm{TAZ}$ to total TAZ at E13.5, E15.5, and E18.5. (e) Immunofluorescence localization of SMG TAZ (green) at E13.5, E15.5, and E18.5, counterstained with F-actin (red). (f) Immunofluorescence localization of TAZ (green) at E15.5, counterstained with nuclei (blue).

expression of SMGs treated with silencing Lats2 siRNA (S) to non-silencing siRNA (NS). Statistical analysis was performed using an unpaired sample $t$-test comparing
mRNA expression levels of Lats2 in NS controls with Lats2 siRNA-treated glands from three different RNA preparations. 
f

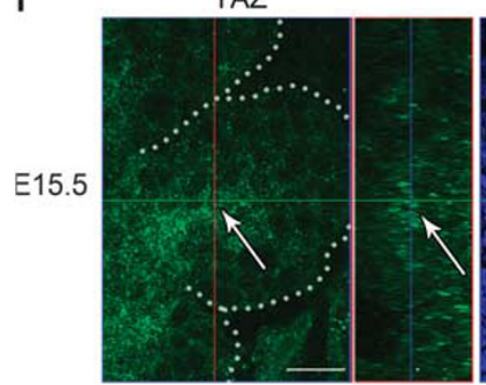

nuclei

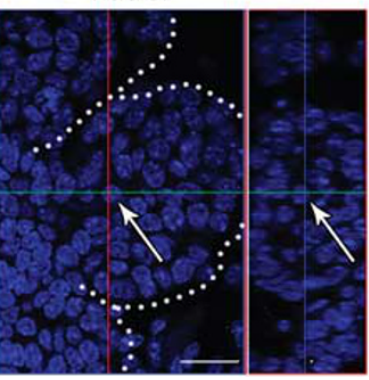

merge

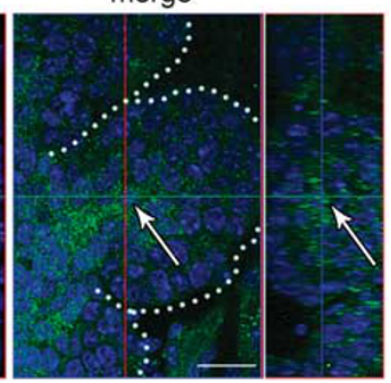

Figure 1 (Continued)

\section{Statistics}

For immunoblot and co-IP assays, mean values from a minimum of triplicate samples are reported \pm s.e.m. All data were analyzed using unpaired sample $t$-tests. Values of $P<0.05$ were considered significant.

\section{RESULTS}

\section{Expression and Localization of the Hippo Pathway Effector TAZ During SMG Embryonic Development}

Mouse SMG development is initiated at embryonic day 11 (E11) as an epithelial thickening that gives rise to a bud structure by E12.5 (Figure 1a, E12.5). During morphogenesis, the initial bud grows and expands into the underlying mandibular mesenchyme, whereas cells in the stalk region reorganize to form a presumptive duct. The expansion of the bud into the mesenchyme is accompanied by repetitive clefting and new bud formation, which is coordinated with the extension of the primary duct into an array of secondary ducts leading into the newly forming terminal buds. Throughout this process, regions of the developing salivary epithelium undergo cytodifferentiation to produce a tree-like structure by E18.5 consisting of differentiated terminal secretory units, the acini, connected to an array of ducts that empty into the principal excretory duct and the oral cavity (Figure 1a, E18.5). The SMG undergoes another round of morphogenesis and cytodifferentiation during the postnatal period to give rise to an adult gland 3 weeks after birth.

Accurate assembly and regulation of E-cadherin junctions are critical for the cytodifferentiation of SMGs. ${ }^{15,16,27,28}$ As E-cadherin-mediated cell adhesion directs the localization and activity of the Hippo pathway effectors TAZ and YAP, ${ }^{18,22}$ we sought to determine whether Hippo signaling had a role in SMG development at three embryonic stages representing early morphogenesis (E13.5), initial cytodifferentiation (E15.5), and late cytodifferentiation (E18.5). At these stages, E-cadherin junctions undergo dynamic changes in organization, with increasing levels of associated $\alpha$-catenin indicating junction maturation. ${ }^{16}$ Further, TAZ and YAP transcripts are detected at these embryonic stages of development (http://sgmap.nidcr. nih.gov/sgmap/sgexp.html), with TAZ transcripts displaying high abundance. Therefore, to investigate changes in Hippo pathway activity during SMG embryonic development, we focused our initial analysis on TAZ expression and phosphorylation. Immunoblot analyses showed that the abundance of TAZ increased with cytodifferentiation (Figure $1 \mathrm{~b},{ }^{\star} P<0.05$ ). This was associated with progressively higher levels of phospho-TAZ (P-TAZ) from E13.5 to E18.5 (Figure 1c, $\left.{ }^{\star} P<0.05\right)$. Comparison of the ratios of phospho-TAZ with total TAZ revealed an increase in the phosphorylation levels at later developmental stages (Figure 1d). Thus, cytodifferentiation of SMGs is accompanied by increased levels and phosphorylation of TAZ, suggesting that Hippo pathway signaling has a role in the establishment and/or maintenance of the acinar and ductal cell polarity.

To further examine the roles of TAZ in SMG morphogenesis, we followed the expression and localization of TAZ at different stages of embryonic development by immunofluorescence localization coupled with confocal microscopy. Our previous studies identified two populations of salivary cell progenitors early in SMG morphogenesis: an outer layer of polarized acinar progenitors and a population of ductal progenitors in the inner bud regions. ${ }^{16}$ Interestingly, TAZ was differentially distributed in acinar and ductal progenitors early in morphogenesis at E13.5. In the polarized pro-acinar cell layer in contact with the basement membrane, which contains well-organized E-cadherin junctions, TAZ was found at the cell-cell borders (Figure 1e, E13.5, top insets, arrows), suggesting that TAZ is associated with E-cadherin junctions in these cells. We have also shown that during SMG morphogenesis, the acinar progenitors surround a population of proliferating polymorphous ductal progenitors with poorly organized E-cadherin junctions in the distal regions of the terminal buds, whereas the proximal region of the glands contain cells already forming secondary ductal structures. These secondary ductal structures extend the primary duct from the stalk region into the newly forming terminal buds, a process during which a subset of immature ductal progenitors cease to proliferate and undergo reorganization into ductal structures through cytoskeletal rearrangements as delineated by robust staining of F-actin. ${ }^{16}$ Examination of the distribution of TAZ in the inner bud region comprising ductal progenitors revealed that TAZ was predominantly cytoplasmic, with an exception for a fraction localized to the 
a

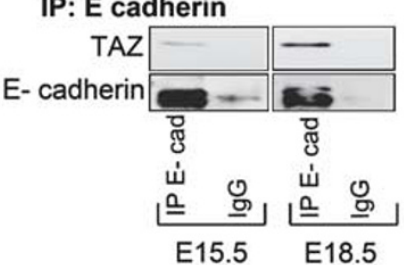

C
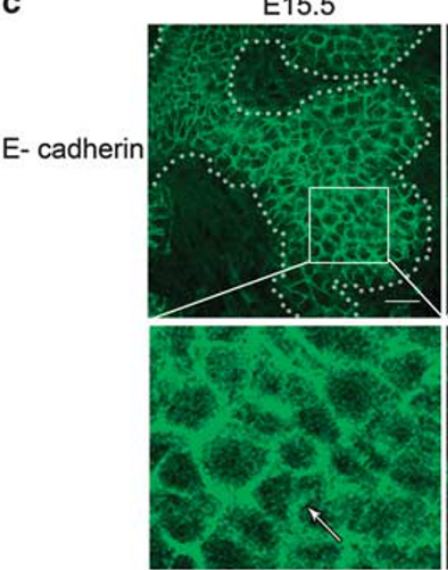

e
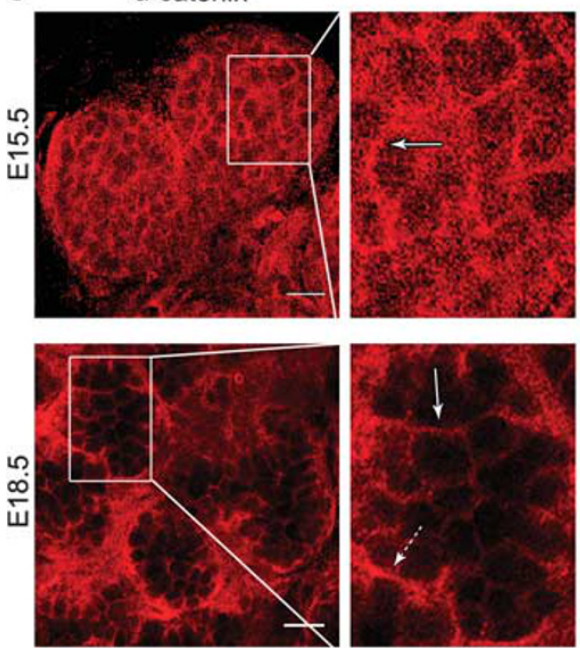

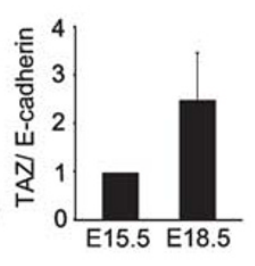

E18.5

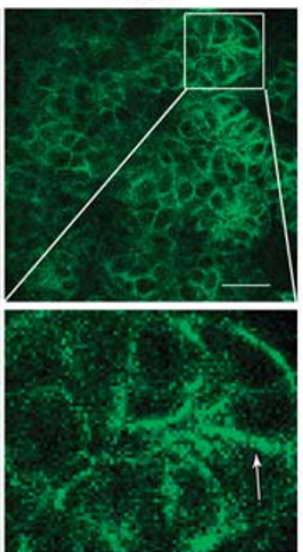

b

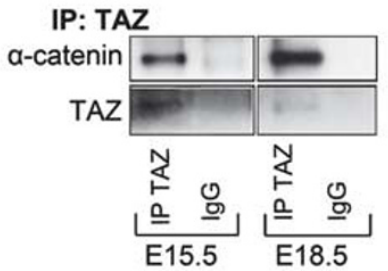

d
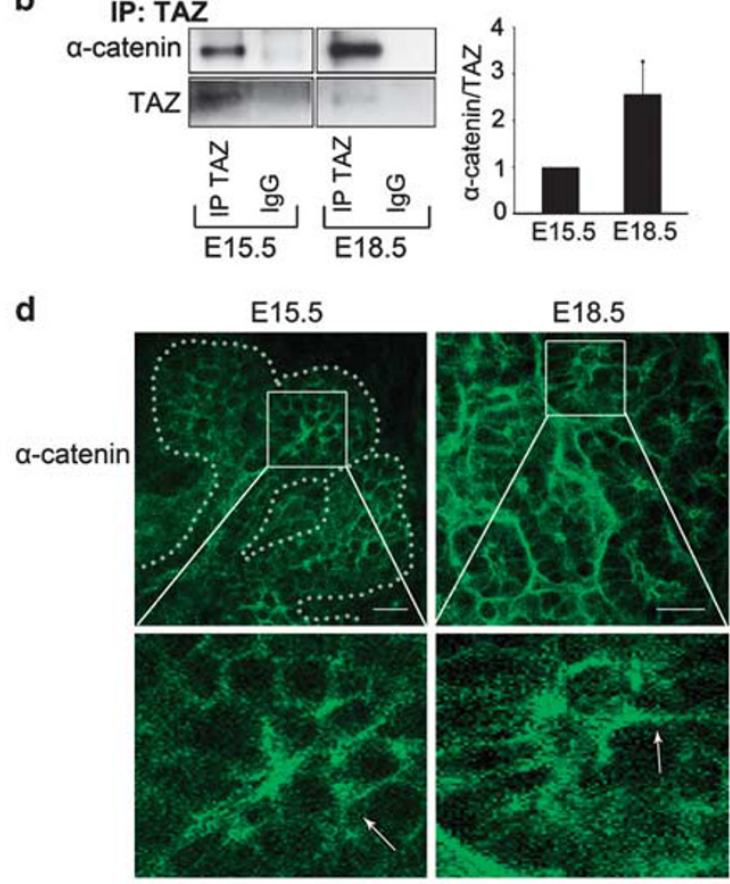

TAZ
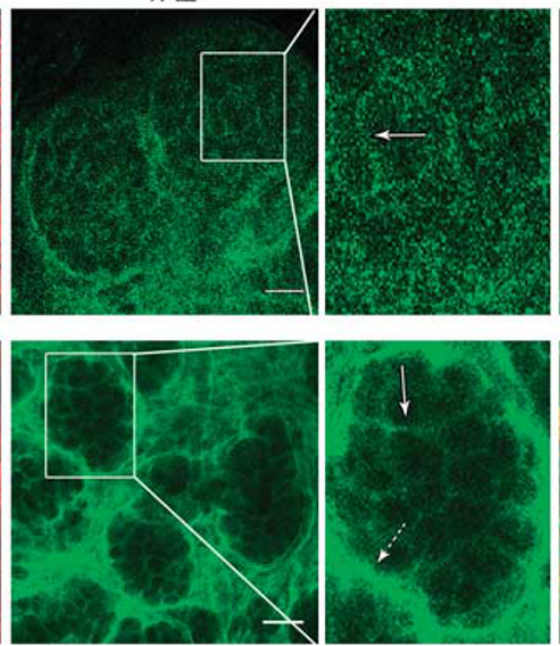

merge
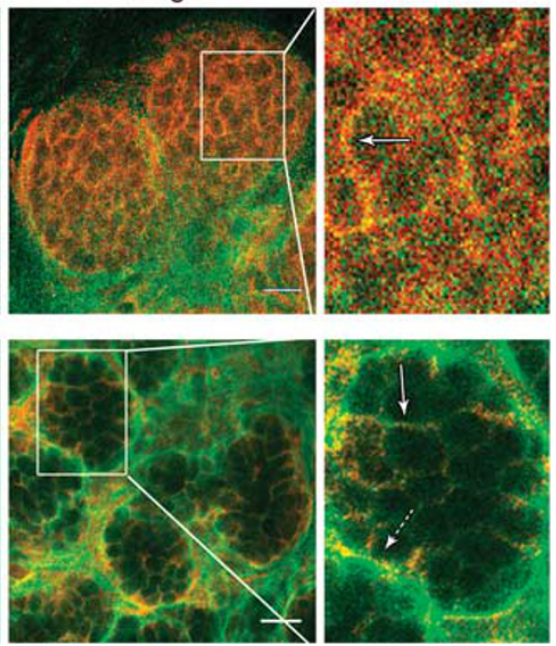

Figure 2 TAZ interacts with E-cadherin junctional complexes throughout development. (a) E-cadherins were immunoprecipitated from E15.5 and E18.5 salivary submandibular gland (SMG) tissue lysates and their interaction with TAZ was assessed by immunoblot. Bar graph, quantification of TAZ/Ecadherin levels. (b) TAZ was immunoprecipitated from E15.5 and E18.5 SMG tissue lysates, and its interaction with $\alpha$-catenin was examined by immunoblot. Bar graph, quantification of $\alpha$-catenin/TAZ levels. (c) Immunofluorescence localization of E-cadherin at E.15.5 and E18.5. Size bar: $20 \mu$ m. (d) Immunofluorescence localization of $\alpha$-catenin at E.15.5 and E18.5. Size bar: $20 \mu \mathrm{m}$. (e) Immunofluorescence colocalization of $\alpha$-catenin (red) and TAZ (green) at E15.5 and E18.5. Size bar: $20 \mu \mathrm{m}$.

sites of new duct formation as marked by F-actin in the proximal bud regions (Figure 1e, E13.5, arrows, lower insets). In addition, TAZ was detected in the mesenchyme surrounding the epithelial buds.

During morphogenesis at E15.5, the acinar progenitors continue to expand and ultimately form unit acinar structures by the end of cytodifferentiation at E18.5. Development of the ductal tree moves from the proximal to distal regions of the terminal buds and involves the specification of apical membranes by F-actin and interacting components. ${ }^{16}$ Immunofluorescence localization of TAZ at E15.5 revealed that it continued to localize at intercellular junctions in the acinar progenitor cells (Figure 1e, E15.5, outer cell layer, arrows). Moreover, TAZ was more prominently defined in the newly forming duct regions, where it colocalized with F-actin (Figure 1e, E15.5, arrows, insets). Interestingly, at this stage, TAZ was present in both the cytoplasm and nucleus (Figure 1f, arrows, $x-y$ and orthogonal, $x-z$ sections). Late in 
a

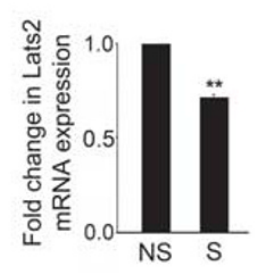

b

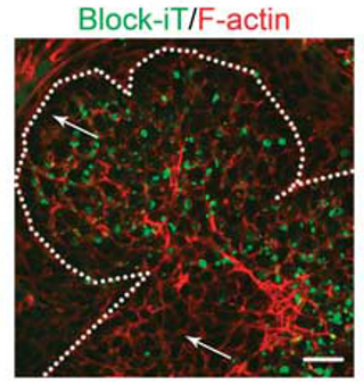

f

NS

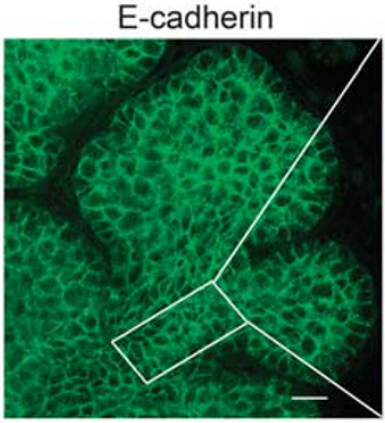

C

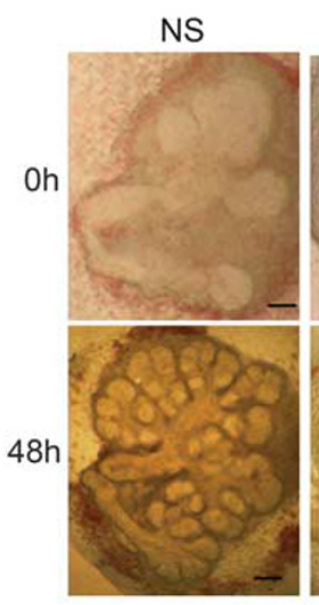

$\mathrm{S}$

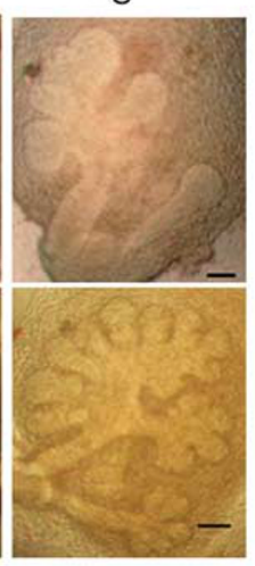

d

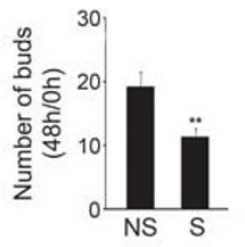

e

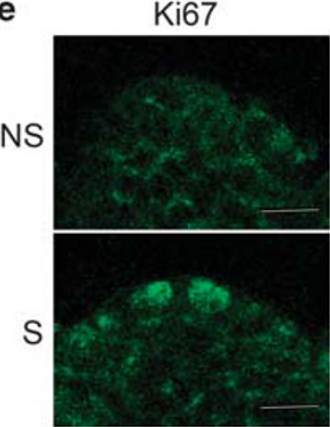

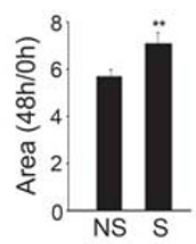

merge

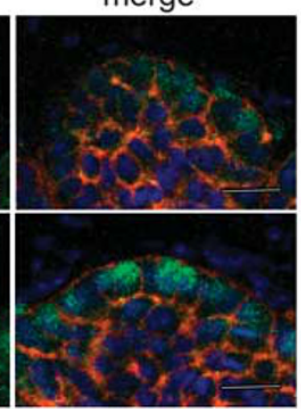

F-actin
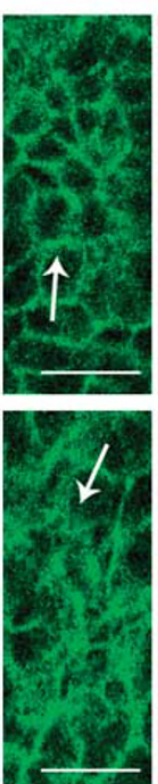

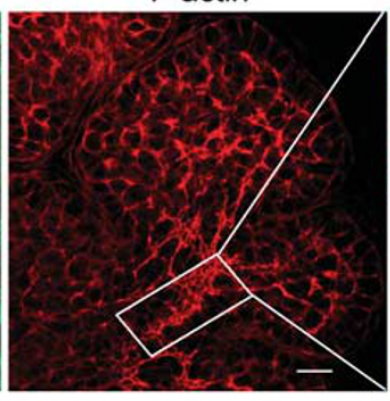

merge
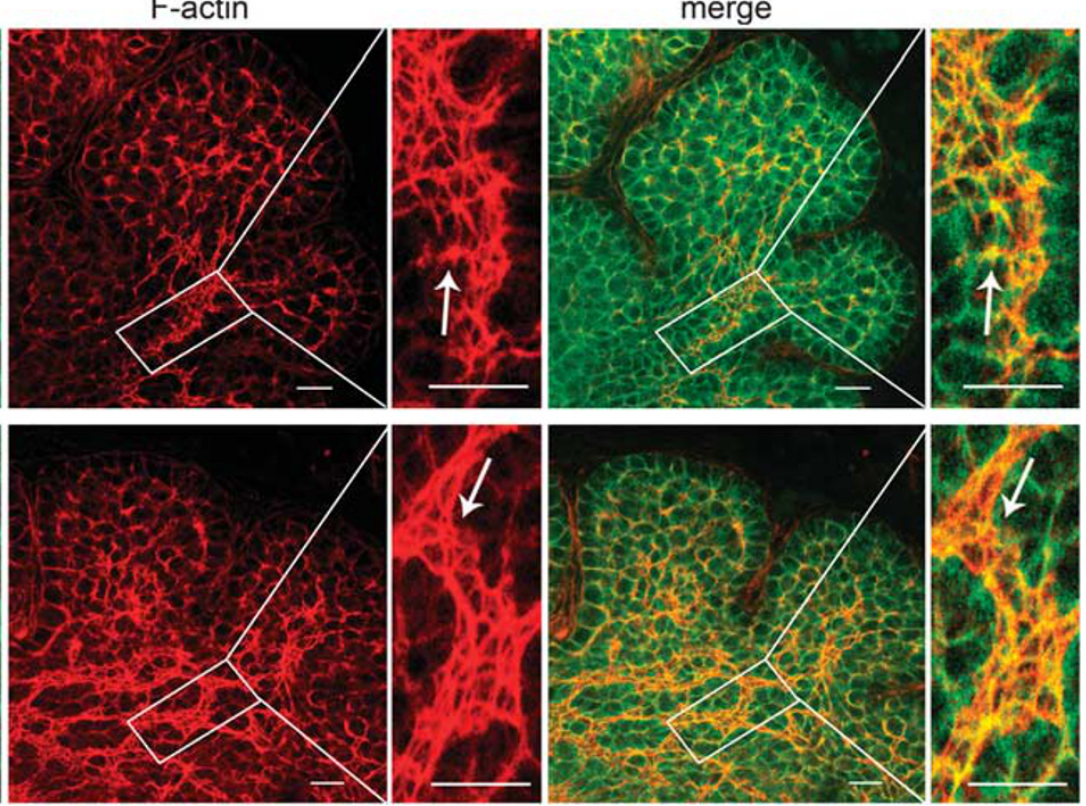

Figure 3 Inhibition of Lats2 kinase perturbs salivary submandibular gland (SMG)-branching morphogenesis. (a) Quantitative PCR of Lats2 transcript levels in non-silenced (NS) or Lats2-silenced (S) glands. ${ }^{* *} P<0.01$. (b) Immunofluorescence localization of BLOCK-iT Fluorescent Oligo, a marker of transfection efficiency (green) counterstained with F-actin (red). (c) Phase microscopy images of NS and S SMGs transfected at stage E13.5 at 0 and $48 \mathrm{~h}$ after Lats2 inhibition. (d) Morphometric analyses of the number of buds at $48 \mathrm{~h} / 0 \mathrm{~h}$ (top) and area of buds at $48 \mathrm{~h} / 0 \mathrm{~h}$ (bottom) of NS and S SMGs. ${ }^{*} P<0.01$. (e) Immunofluorescence localization of Ki67 (green), counterstained with F-actin (red) and nuclei (blue), in NS and S glands. Size bar: $20 \mu \mathrm{m}$. (f) Immunofluorescence localization of E-cadherin (green), counterstained with F-actin (red), in NS and S glands. Size bar: $20 \mu \mathrm{m}$. (g) Immunofluorescence localization of TAZ (green), counterstained with F-actin (red), in NS and S glands. Size bar: $20 \mu \mathrm{m}$.

embryonic cytodifferentiation at E18.5, TAZ was organized at intercellular junctions in differentiated acinar and ductal cells (Figure 1e, E18.5, arrows, insets). We note that a significant fraction of TAZ was localized to sites representing either the basal regions of the SMG epithelium and/or the adjacent myoepithelial and mesenchymal compartments. Collectively, these data show that SMG development is associated with increased levels of phosphorylated and junctional TAZ in differentiating acinar and ductal cells.

\section{TAZ Interacts with E-Cadherin Junctional Complexes in a Development-Dependent Manner}

Hippo pathway signaling is induced by cadherin-based adhesion signals, with TAZ and YAP binding to E-cadherin and $\alpha$-catenin promoting TAZ and YAP cytoplasmic localization. ${ }^{25,29,30}$ Therefore, we investigated whether increased TAZ at cell-cell borders during SMG cytodifferentiation reflects increased binding to E-cadherin and/or $\alpha$-catenin. co-IP studies revealed that TAZ was present in E-cadherin 
g
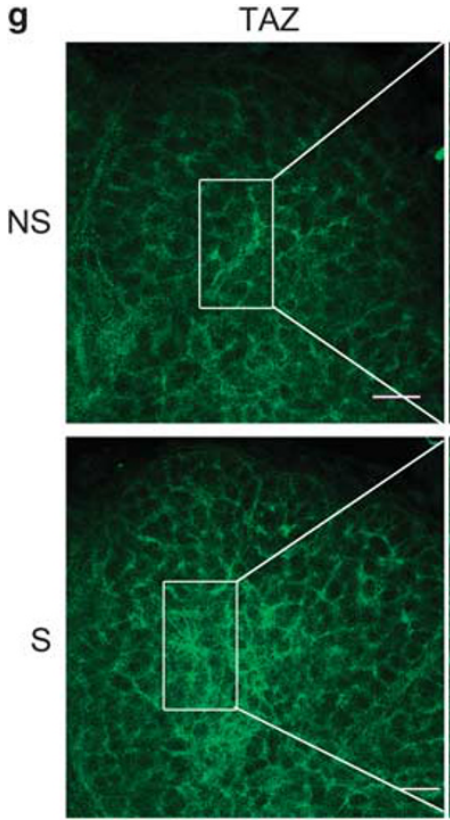

F-actin
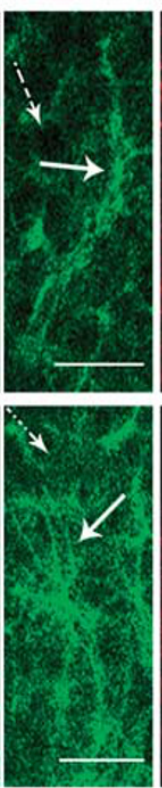
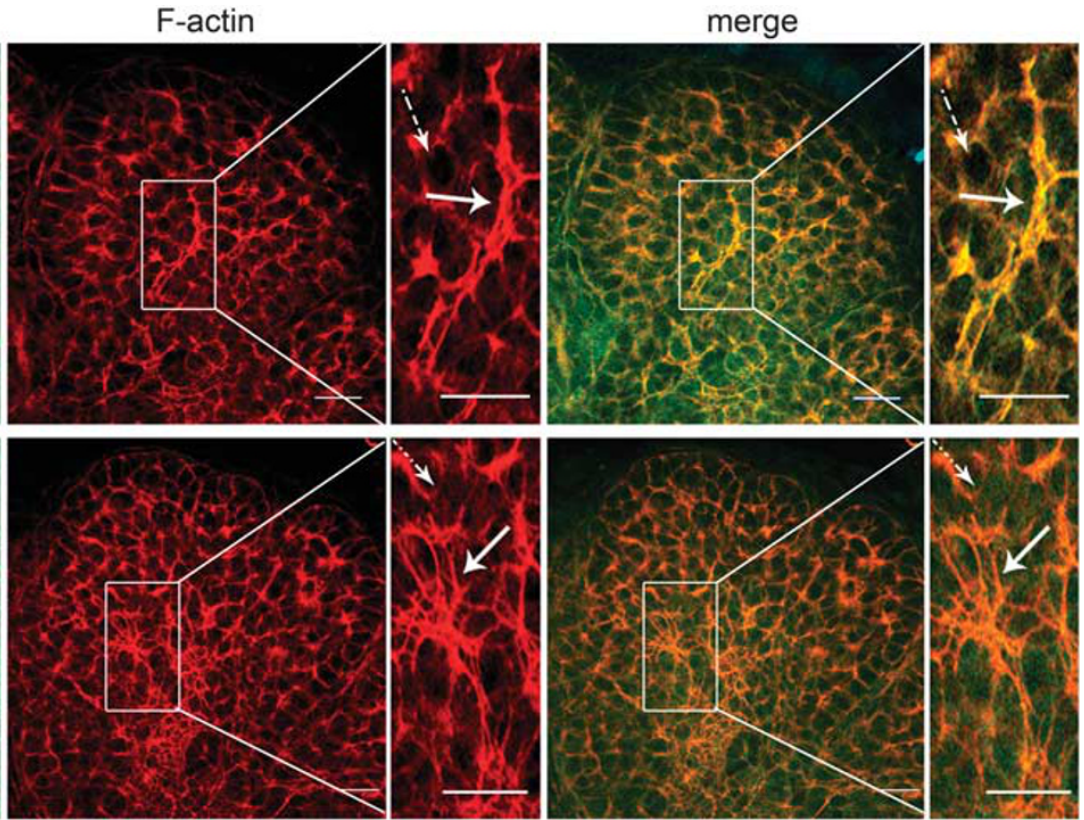

Figure 3 (continued)

complexes at E15.5, and that its association with E-cadherin increased at E18.5 (Figure 2a). This coincided with a more focused organization of E-cadherin at cell-cell-cell borders at E18.5, previously shown to indicate mature AJs (Figure 2c, arrows, insets). ${ }^{14,20}$ Similarly, co-IP of TAZ complexes revealed that TAZ associated with augmented levels of $\alpha$ catenin at E18.5 compared with E15.5 (Figure 2b), which coincided with increased recruitment of $\alpha$-catenin to cell-cell borders (Figure 2d, arrows, insets). We have previously shown that increased presence of $\alpha$-catenin at the membrane reflects its association with mature E-cadherin junctions. ${ }^{16}$ Further, immunofluorescence analysis of SMGs doubly stained for TAZ and $\alpha$-catenin revealed increased colocalization from E15.5 to E18.5 (Figure 2e, arrows, insets). Thus, as E-cadherin junctions mature, TAZ associates with E-cadherin/ $\alpha$-catenin complexes, suggesting that TAZ has a role in E-cadherin junction maturation during SMG morphogenesis.

\section{Inhibition of the Hippo Pathway Lats2 Kinase Perturbs SMG-Branching Morphogenesis}

Activation of Lats kinases leads to phosphorylation and subsequent nuclear exclusion of TAZ and YAP, whereas inhibition of Lats kinase activity reduces TAZ and YAP phosphorylation, resulting in increased TAZ and YAP nuclear localization. ${ }^{21}$ Lats2 expression is high in developing SMGs (http://sgmap. nidcr.nih.gov/sgmap/sgexp.html). Thus, to further assess the role of Hippo signaling in SMG development, we examined the consequences of inhibiting Lats2 kinase in E13.5 ex vivo organ cultures on branching morphogenesis.

Treatment of E13.5 SMG organ cultures with siRNA to Lats2 kinase for $48 \mathrm{~h}$ resulted in a 30\% reduction in Lats2 transcript levels in silenced (S) glands compared with nonsilenced (NS) controls (Figure 3a). In these studies, we also followed the uptake of siRNA by Block-iT, a small fluorescein-tagged oligonucleotide (Figure 3b). Results showed that the polarized outer acinar progenitor cell layer and the differentiating duct cells in the stalk region did not label extensively with Block-iT, suggesting that Lats2 siRNAmediated knockdown may not be efficient in these cells (Figure 3b, arrows). In contrast, the ductal progenitors, including the reorganizing cells in the proximal bud regions, displayed a robust staining for Block-iT, suggesting that siRNA-mediated knockdown of Lats2 predominantly occurred in these cells (Figure 3b). This differential uptake of Block-iT by cells in the developing SMG may explain the observed modest decrease in Lats 2 mRNA levels following siRNA treatment. Nonetheless, even a 30\% downregulation of Lats 2 mRNA coincided with distinct morphological changes that included a flattened central area in the main branching region, larger end buds, and diminished overall branching (Figure $3 \mathrm{c}$ ). The flattening of the glands reflects inhibition of ductal extensions, most likely because of the failure of cells in the duct-forming regions to differentiate, which is required for subsequent cellular rearrangements, consistent with the proliferationpromoting activity of TAZ/YAP. Indeed, morphometric measurements revealed that partial inhibition of Lats2 kinase leads to a significant increase in the tissue surface area and a reduction in the number of buds (Figure 3d, $\left.{ }^{*} P<0.01\right)$. Although Ki67 staining of cells in the reorganizing duct region was not easily discernible, the Lats2-inhibited glands displayed increased proliferation in the outer layer (Figure 3e). 
a
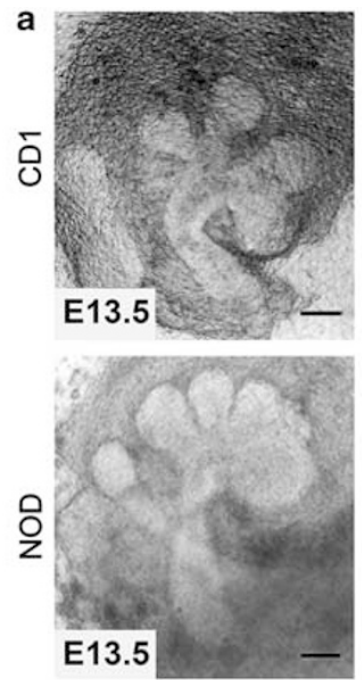
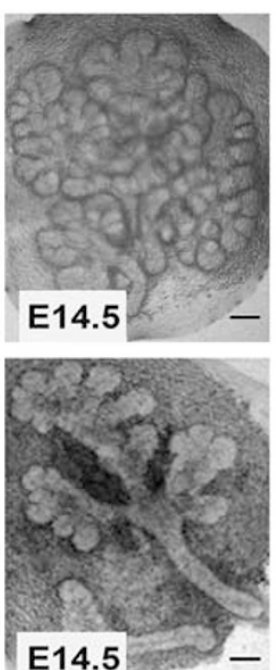
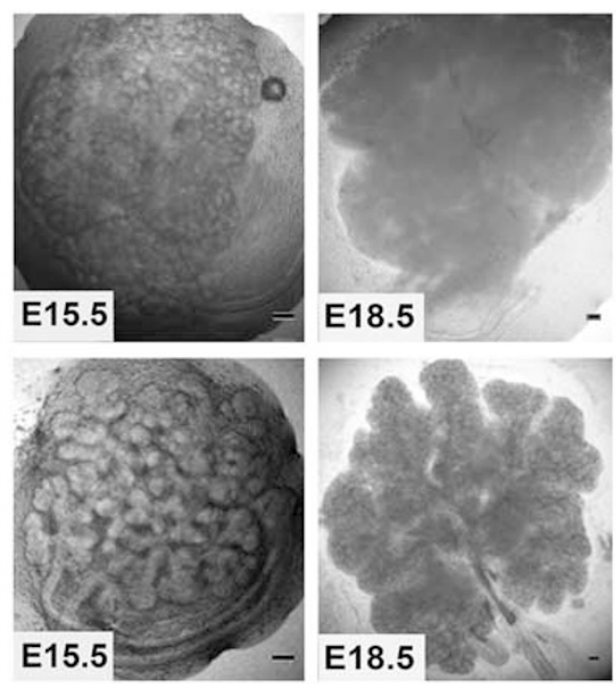

b

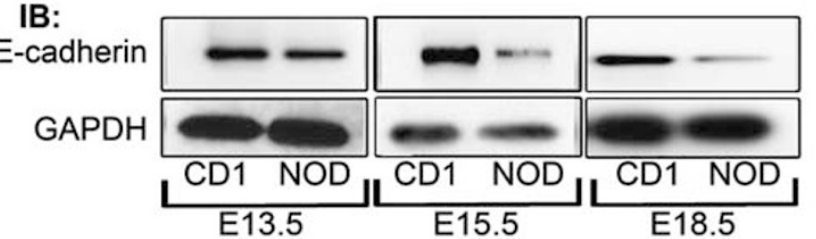

C

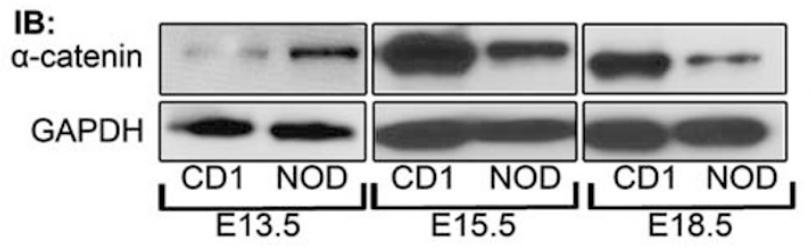

d

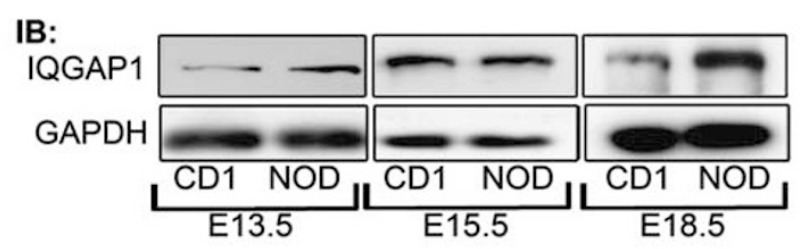

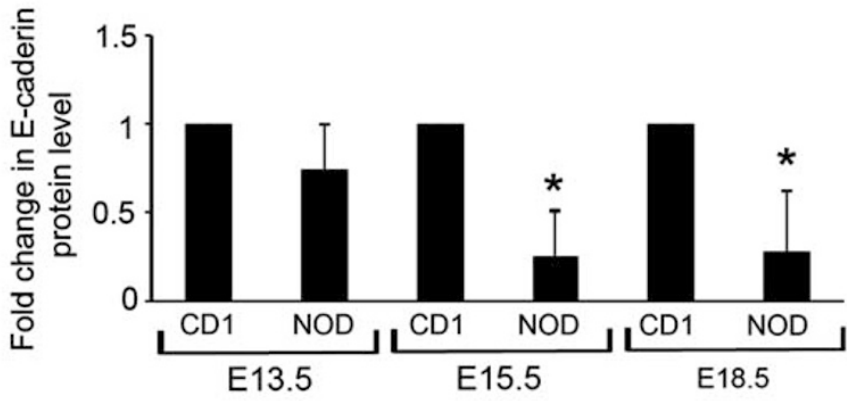
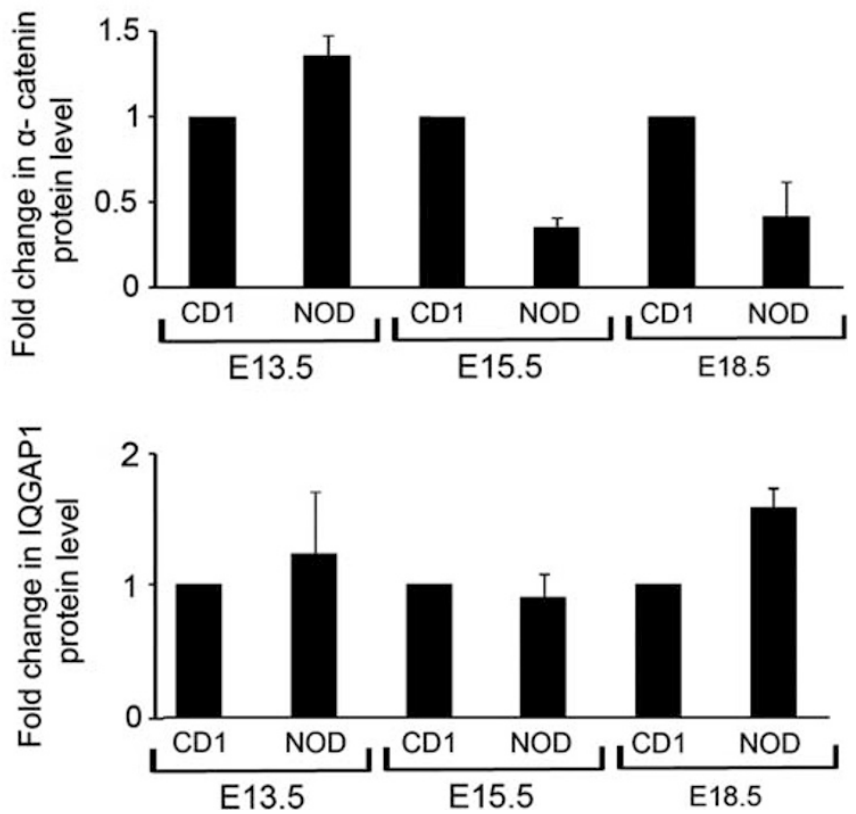

Figure 4 Non-obese diabetic (NOD) mouse salivary submandibular glands (SMGs) display dysmorphogenesis and altered expression levels of TAZ and E-cadherin junctional components. (a) Phase microscopy images of SMGs isolated from NOD mice on embryonic days E13.5-E18.5 during SMGbranching morphogenesis. Size bar: $20 \mu \mathrm{m}$. (b) Immunoblots comparing E-cadherin expression levels in CD1 and NOD SMGs at E13.5, E15.5, and E18.5. Bar graph, fold change in E-cadherin levels after normalization to glyceraldehyde 3-phosphate dehydrogenase (GAPDH), ${ }^{*} P<0.05$. (c) Immunoblots assessing $\alpha$-catenin expression levels in CD1 and NOD SMGs at E13.5, E15.5, and E18.5. Bar graph, fold change in $\alpha$-catenin levels after normalization to GAPDH. (d) Immunoblots comparing IQGAP1 expression levels in CD1 and NOD SMGs at E13.5, E15.5, and E18.5. Bar graph, fold change in IQGAP1 levels after normalization to GAPDH. (e) Immunoblots comparing TAZ expression levels in CD1 and NOD SMGs at E13.5, E15.5, and E18.5. Bar graph, fold change in TAZ levels after normalization to GAPDH, ${ }^{*} P<0.05$. (f) Immunoblots comparing $\mathrm{p}-\mathrm{TAZ}$ expression levels in CD1 and NOD SMGs at E13.5, E15.5, and E18.5. Bar graph, fold change in $p-T A Z$ levels after normalization to GAPDH, ${ }^{*} P<0.05$. 
e

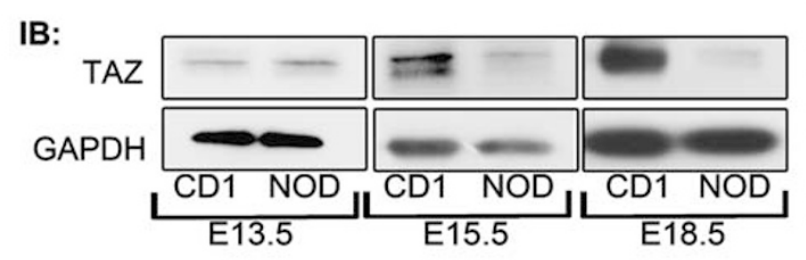

f

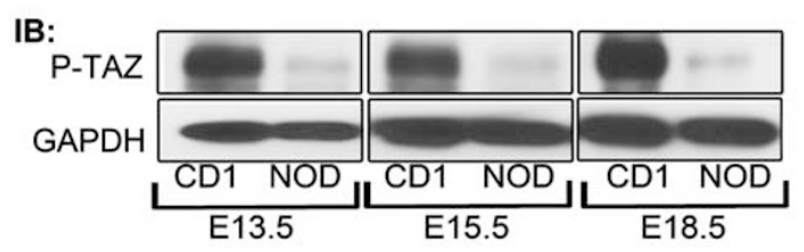

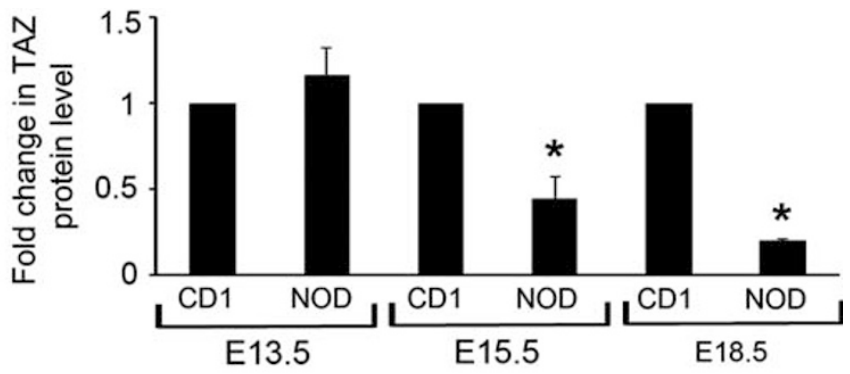

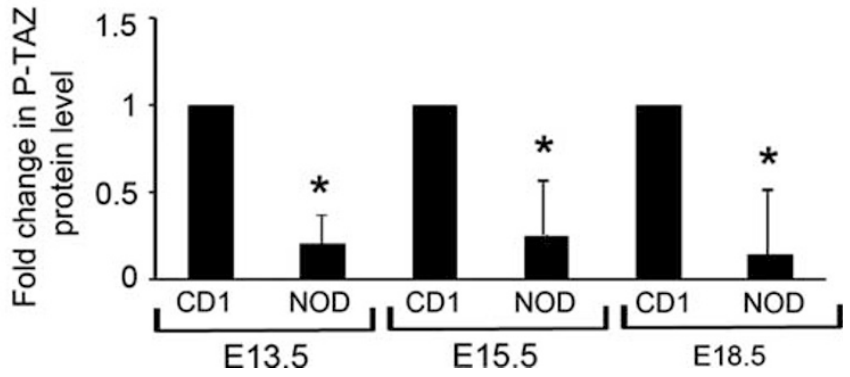

Figure 4 (Continued)

Our previous studies have demonstrated that F-actin demarks newly forming ductal structures in proximal areas of terminal buds. ${ }^{16}$ Although the sites of new duct formation contained tight F-actin cables in NS buds, in the Lats2inhibited glands some regions exhibited dispersed patterns of F-actin distribution in the proximal branching regions (Figure 3f, arrows; F-actin, NS, and S). Such expanded patterns of F-actin distribution in Lats2-inhibited glands suggested that in the absence of Hippo signaling, duct initiation occurred but its extension did not proceed normally. Therefore, Hippo signaling is necessary for proper secondary duct development during SMG-branching morphogenesis.

We have previously reported that during duct development, E-cadherin is well organized at the apical-lateral surfaces of differentiating duct cells and serves as a survival signal. ${ }^{16}$ In Lats2-silenced glands, E-cadherin displayed altered organization in the prospective duct regions (Figure 3f, arrows; E-cadherin, NS, and S). Similarly, although in NS glands TAZ exhibited a well-defined and focused colocalization with F-actin at the apical domains of newly forming duct regions, in Lats2-silenced glands its organization was irregular and spread out, although it still followed the patterns of F-actin (Figure 3f, arrows; TAZ and F-actin, merge, NS, and S). This suggested that cells in which Lats2 was inhibited did not undergo reorganization into secondary ductal structures, thus failing to extend the ductal axes into the expanding gland. Further, the staining of TAZ in Lats2-inhibited glands was more diffuse in cells destined for reorganization, suggesting that TAZ was re-localized from junctions to the nucleus (Figure 3g, dashed arrows). Collectively, these data showed that Hippo pathway Lats2 kinase has a crucial role in secondary duct formation during SMG-branching morphogenesis.

\section{SMGs from NOD Mice Display Dysmorphogenesis Coupled with Altered Expression of TAZ and E-Cadherin Junctional Components}

The NOD mouse has been used extensively as a model of SS, as the diseases in mice show many similarities to the human disease in terms of immunology, affected organs, and gender predisposition. ${ }^{31}$ Given our observations linking the Hippo pathway to the salivary gland development, we next investigated the expression of TAZ in NOD mice, as this mouse model is known to have structural defects in several organs and to develop SS-like disease. ${ }^{8}$ We first examined the gross morphology of SMGs from NOD mice and compared them with SMGs from wild-type, CD1, mice. Already at E13.5, SMGs from NOD mice displayed a broader initial branching region, and at E14.5 they exhibited a significantly more flattened morphology with less branching than the wild-type glands, resembling Lats2-silenced glands (Figure 4a). At E15.5, NOD SMGs had larger terminal buds, and they lacked the three-dimensional budding pattern typical of wild-type glands. These altered morphological features and growth characteristics were also evident at E18.5, with NOD glands displaying less compact morphology and diminished branching (Figure 4a).

Examination of E-cadherin junctional components in NOD SMGs revealed dramatic changes in their abundance at all three stages of embryonic morphogenesis. E-cadherin levels were significantly decreased in developing NOD glands at stages E15.5 and E18.5 (Figure 4b), and although the levels of $\alpha$-catenin were higher in E13.5 NOD glands compared with their wild-type counterparts, they declined with cytodifferentiation at E15.5 and E18.5 (Figure 4c). Interestingly, IQGAP1 was more prominent in NOD SMGs at E18.5, suggesting that diminished E-cadherin adhesion is associated 

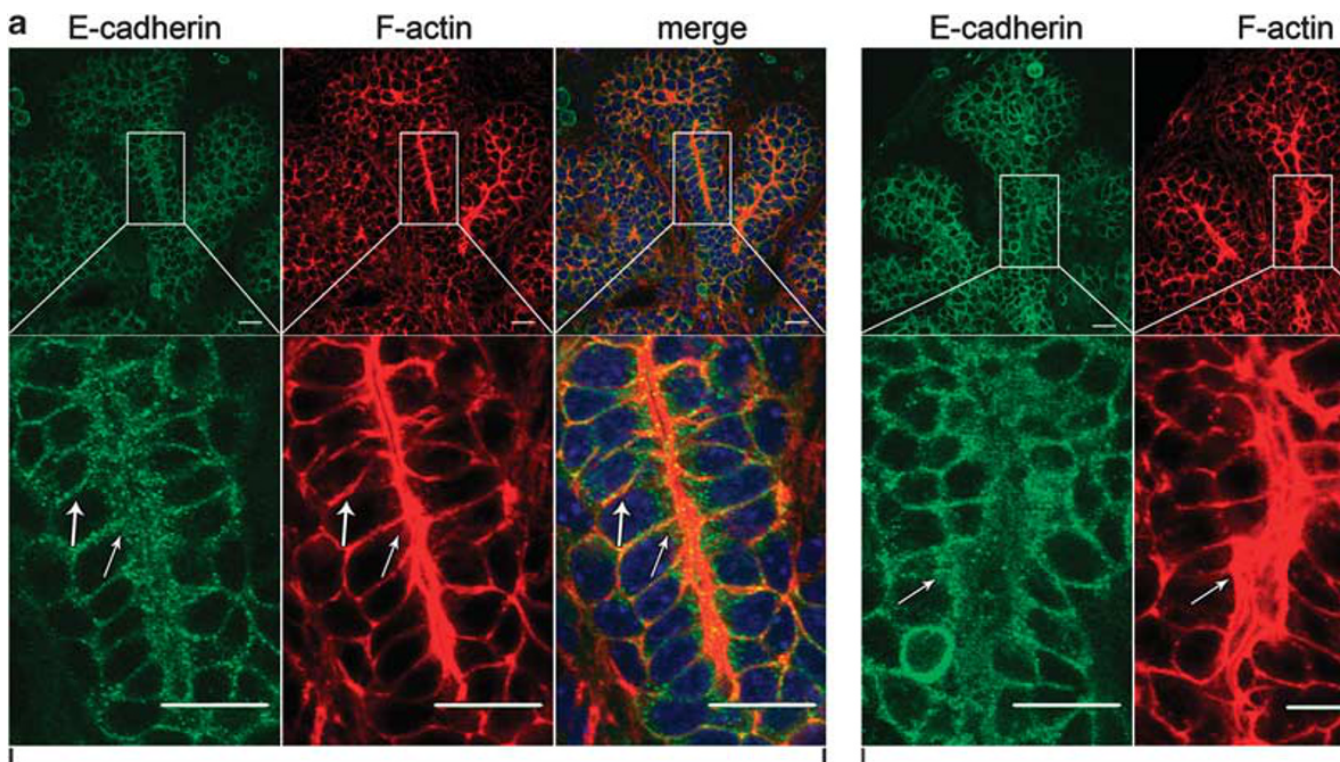

\section{CD1}

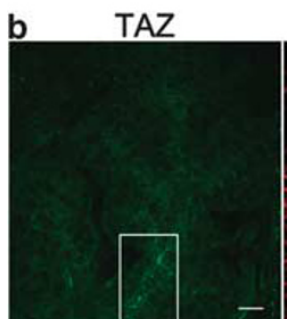

F-actin

merge

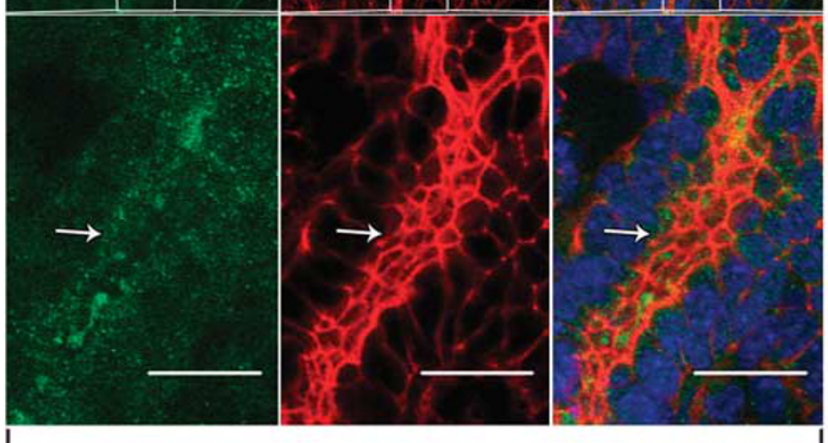

CD1
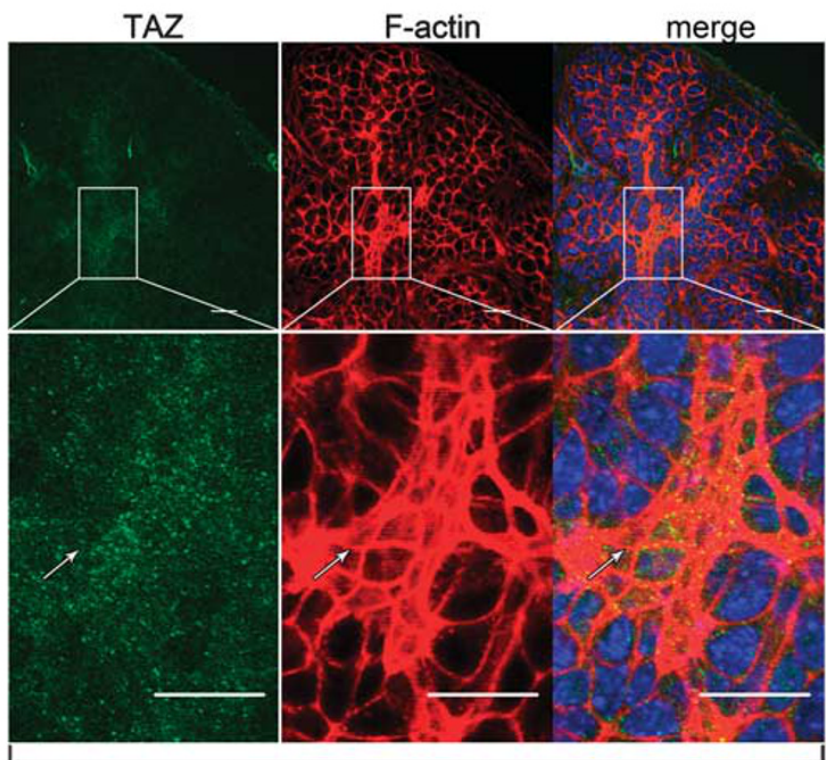

NOD

Figure 5 Non-obese diabetic (NOD) salivary submandibular glands (SMGs) have aberrant duct formation and disorganized E-cadherin and TAZ. (a) Immunofluorescence localization of E-cadherin (green) in CD1 and NOD mouse SMGs at stage E15.5, counterstained with F-actin (red). Size bar: $20 \mu \mathrm{m}$. (b) Immunofluorescence localization comparing TAZ (green) in CD1 and NOD mouse SMGs at stage E15.5, counterstained with F-actin (red). Size bar: $20 \mu \mathrm{m}$.

with increased expression of this junction destabilizer (Figure 4d). ${ }^{32}$ The most dramatic changes in NOD SMGs, however, were detected for TAZ and its phosphorylated species. Although at E13.5, levels of TAZ were similar between wild-type and NOD SMGs, they were greatly diminished at later stages of cytodifferentiation in the NOD glands. This pattern was also observed for the phosphorylated form of TAZ, with a dramatic reduction in P-TAZ in NOD glands already apparent at E13.5 (Figures $4 \mathrm{e}$ and $\mathrm{f}$ ). Loss of phospho-TAZ early in morphogenesis correlated with the defective branching morphology of E13.5 NOD SMGs. These findings further support a role for TAZ in proper morphogenesis and cytodifferentiation of the SMG.

\section{NOD SMGs Exhibit Defects in Duct Formation}

As expression levels of E-cadherin and $\alpha$-catenin are greatly diminished in embryonic NOD SMGs undergoing cytodifferentiation, it was not possible to assess the interactions between TAZ and E-cadherin junctions by co-IP. To gain insight into the quality of E-cadherin junctions in NOD SMGs, we investigated E-cadherin localization by indirect 


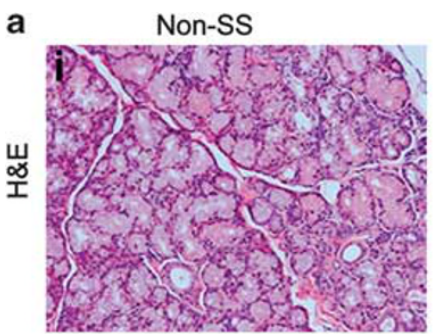

b
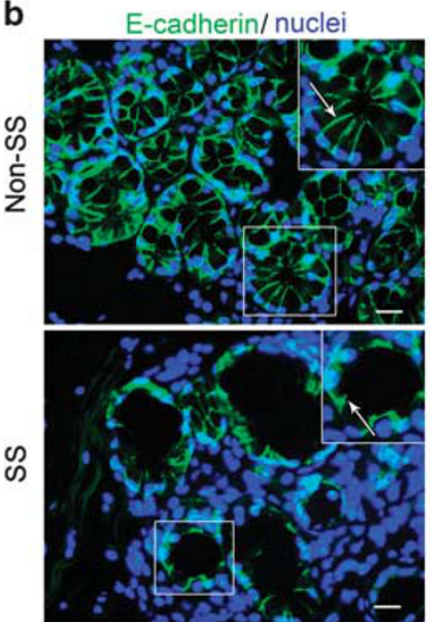

f
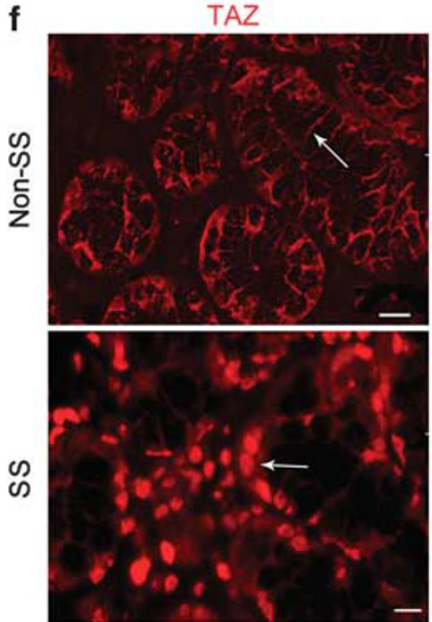

SS- Focus score 1

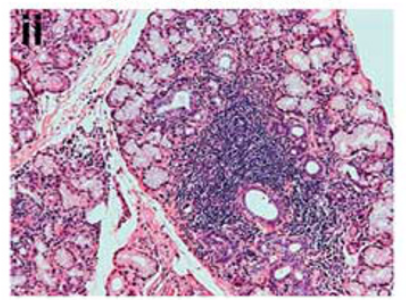

C $\quad \beta$-catenin/ nuclei
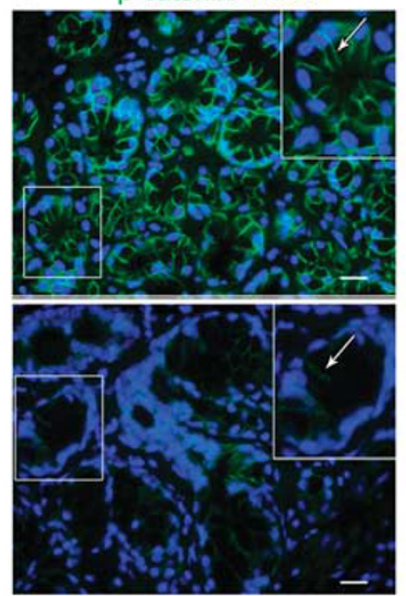

TAZI nuclei
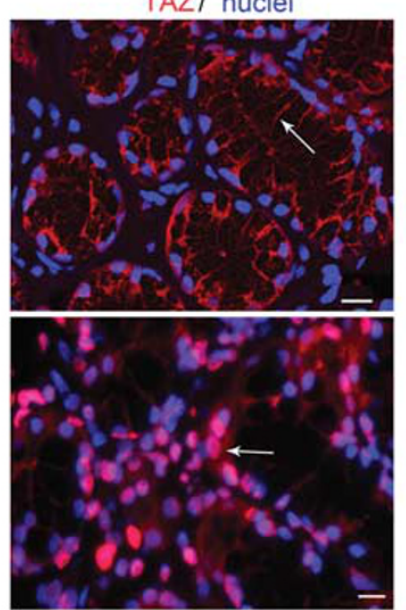

SS- Focus score 1

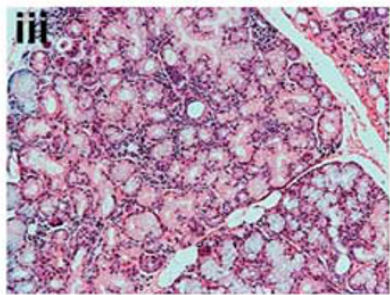

d a-catenin/ nuclei
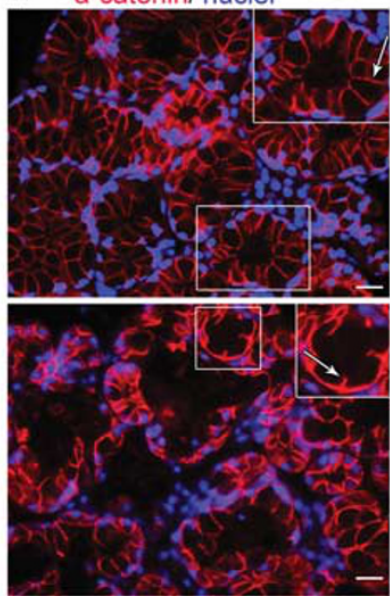

g
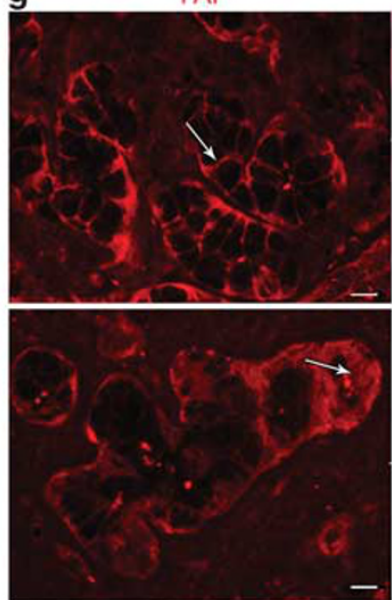

SS- Focus score 5

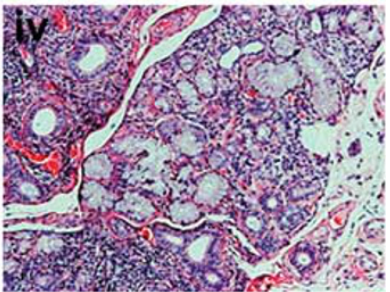

e IQGAP1/ nuclei
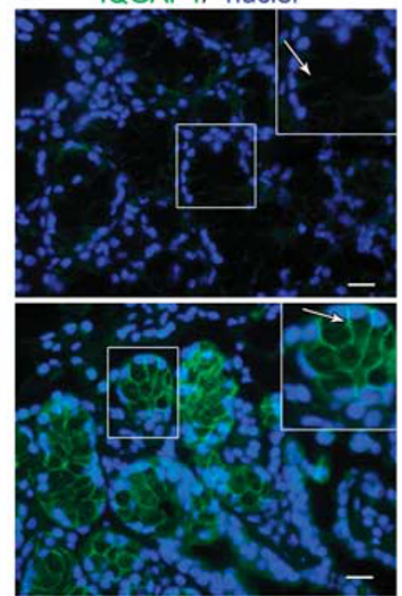

YAPI nuclei
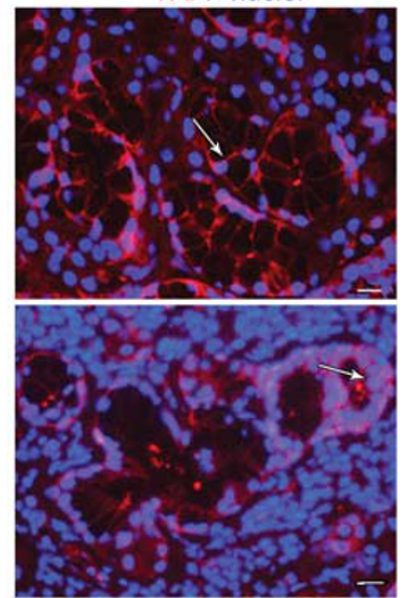

Figure 6 Sjogren's syndrome (SS) compatible labial gland biopsies display loss of polarity, dysregulated Hippo signaling, and extracellular matrix (ECM) remodeling. (a) Hematoxylin and eosin (H\&E) staining of a biopsy from an SS non-compatible (non-SS) patient compared with different degrees of lymphocytic infiltrations (measured as the focus score) of SS patients. Size bar: $50 \mu \mathrm{m}$. (b) Immunofluorescence localization of E-cadherin in SS and nonSS glands, counterstained with 4',6-diamidino-2-phenylindole (DAPI). Size bar: $20 \mu \mathrm{m}$. (c) Immunofluorescence analyses of $\beta$-catenin in SS and non-SS glands, counterstained with DAPI. Size bar: $20 \mu \mathrm{m}$. (d) Immunofluorescence localization of $\alpha$-catenin in SS and non-SS glands, counterstained with F-actin. Size bar: $20 \mu \mathrm{m}$. (e) Immunofluorescence localization of IQGAP1 in SS and non-SS glands, counterstained with DAPI. Size bar: $20 \mu \mathrm{m}$. (f) Immunofluorescence localization of TAZ in SS and non-SS glands (left panel), counterstained with DAPI (right panel). Size bar: $20 \mu \mathrm{m}$. (g) Immunofluorescence localization of YAP in SS and non-SS glands (left panel), counterstained with DAPI (right panel). (h) Immunofluorescence localization of connective tissue growth factor (CTGF) in SS and non-SS glands. Size bar: $20 \mu \mathrm{m}$. (i) Immunofluorescence localization of fibronectin in SS and non-SS glands. Size bar: $20 \mu \mathrm{m}$.

immunofluorescence at E15.5, an embryonic stage at which pronounced differences in the abundance of E-cadherin and $\alpha$-catenin are detected by immunoblots between wild-type and NOD SMGs. Whereas E-cadherin displayed apical-lateral organization in columnar cells in the duct-forming region of wild-type glands, in SMGs from NOD mice, its distribution at the apical-lateral domains was less focused, coincident with the loss of columnar morphology and expansion of duct-forming regions (Figure 5a, arrows, E-cadherin). A similar aberrant pattern of distribution was also found for 

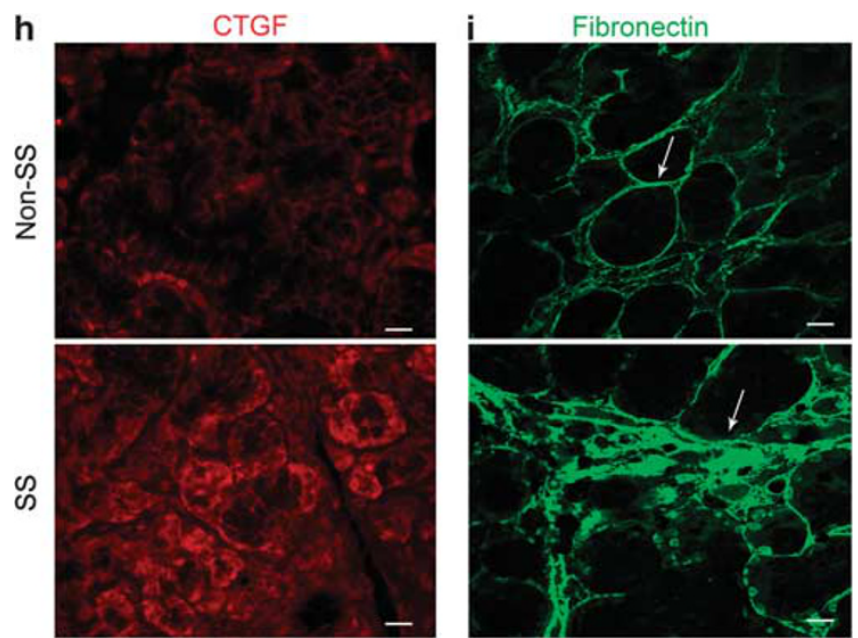

Figure 6 (Continued)

F-actin in NOD SMGs (Figure 5a, arrows, F-actin). Similarly, although a fraction of TAZ was found at the apical domains of cells in the branching ductal region in wild-type glands, its localization was more diffuse in NOD SMGs, which also correlated with disorganized and broader distribution of F-actin (Figure 5b, arrows, TAZ and F-actin). Specifically, although TAZ was detected in the newly forming duct regions in both NOD and wild-type SMGs, in NOD glands the proximal extension of F-actin cables into the bud was compromised. This coincided with expanded and not tightly organized F-actin staining in NOD glands compared with CD1 glands (Figure 5b, arrows, F-actin). Importantly, the disorganization of F-actin in NOD SMGs was similar to that in the Lats2-inhibited glands (Figures $3 \mathrm{f}$ and $\mathrm{g}$ ). The observations that both Lats2-inhibited and NOD SMGs display aberrant duct formation and defects in apical-basal polarity in the reorganizing secondary duct cells suggest that the NOD phenotype is associated with dysregulation of Hippo pathway activity.

\section{Deregulation of Hippo Signaling, Loss of Cell Polarity, and ECM Remodeling are Features of SS}

To determine whether the results from the mouse models are conserved in human SS tissues, we evaluated human labial biopsy specimens from patients with SS for E-cadherin and its junctional binding partners, $\beta$-catenin and $\alpha$-catenin, as well as for the Hippo effectors TAZ and YAP by immunofluorescence analyses and compared them with tissue specimens from SS non-compatible (non-SS) individuals. Hematoxylin and eosin staining of non-SS specimens revealed well-preserved tissue architecture (Figures 6a i). In contrast, SS tissues displayed various degrees of secretory tissue loss and lymphocytic infiltration depending on the severity of the disease, as determined by the focus score (Figure 6a ii-iv). For instance, a biopsy with inflammatory foci evaluated as focus score 1 (Figure 6a ii) was also positive for germinal center (GC)-like structures, reflecting the organization of lymphocytes into well circumscribed foci presenting with a dark and a light zone and thought to be a predictor for lymphoma development. In other areas absent of foci, a pattern of diffusely spread lymphocytic inflammation was seen (Figure 6a iii). The biopsy with a focus score 5 was also GC positive, but in spite of the 'high' focus score and prominent level of infiltration, this gland still maintained tissue integrity (Figure 6a iv). It is important to note that all immunofluorescence imaging was taken from regions distal to the lymphocytic infiltrations to ensure that they did not affect salivary epithelial cells in these analyses (Figure 6a iii).

Immunofluorescence evaluation of non-SS glands from control individuals for E-cadherin and its associated catenins confirmed well-organized E-cadherin junctions in acinar and ductal epithelia (Figures $6 \mathrm{~b}-\mathrm{d}$, arrows; insets, non-SS). In contrast, SS glands showed disrupted distribution of E-cadherin (Figure 6b, arrow, SS). Reduced E-cadherin adhesion in SS specimens was further confirmed by diminished immunofluorescence staining of $\beta$ - and $\alpha$-catenins at lateral borders compared with non-SS controls (Figures $6 \mathrm{c}$ and d, arrows). Concomitant with the loss of $\alpha$-catenin from cell-cell junctions, labial salivary glands from SS patients displayed elevated immunostaining of IQGAP1, known to destabilize E-cadherin adhesion and to function in signaling processes promoting proliferation and extracellular matrix (ECM) remodeling (Figure 6e, arrow). ${ }^{32}$ These structural changes in E-cadherin junctions were associated with deregulation of the downstream effectors of Hippo, TAZ, and YAP. In SS tissues, both TAZ and YAP were lost from lateral cell borders and were instead localized to the basal regions that coincided with the nuclei (Figures $6 \mathrm{f}$ and g, respectively, arrows, SS). This resembled the scenario in NOD SMGs, which displayed upregulation of IQGAP1 (Figure 4d). Significantly, nuclear redistribution of TAZ and YAP in SS specimens correlated with stronger immunostaining of CTGF, a downstream transcriptional target of TAZ and YAP (Figure 6h) and a known regulator of ECM remodeling and inducer of fibrosis. ${ }^{33}$ Moreover, SS patient biopsies exhibited increased deposition of fibronectin, another potential downstream target of TAZ and YAP, confirming ECM remodeling in these tissues (Figure 6i, arrow).

\section{DISCUSSION}

As a complex autoimmune disease, SS has remained recalcitrant to the identification of key factors and processes responsible for its development and pathophysiology. Although defects in the immune system have long been considered to be a cause of SS, recent studies have highlighted loss of cell polarity and structural integrity to have a major role in its etiology. Results presented here further support the hypothesis that loss of salivary cell junctional integrity has a critical role in SS and suggest that the Hippo signaling pathway, in particular, is a key factor in this disease. We provide evidence that a Hippo effector, TAZ, 
interacts with two major determinants of cellular polarity, E-cadherin and $\alpha$-catenin, and that this interaction is required for normal cytodifferentiation and organization of acinar and ductal structures. Significantly, dysregulation of Hippo signaling is a feature of SMGs from NOD mice and of labial specimens from human SS patients, suggesting that defects in Hippo signaling are associated with this disease.

Increasing evidence implicates structural defects as a primary contributor to SS and autoimmunity. In NOD mice, an animal model for SS and type 1 diabetes, regeneration of target organs with exogenous stem cells restores the normal morphology of both SMGs and pancreatic islets, making them resistant to disease. ${ }^{34}$ Earlier studies suggest that the onset of autoimmunity in NOD mice may result from developmental defects, as normally developed SMGs from NODs evade autoimmune exocrinopathy. ${ }^{35}$ In addition, structural defects and hyposalivation in NOD salivary glands can occur independent of lymphocytic infiltration. ${ }^{8,36}$ Recent findings from patients with SS also indirectly support the premise that the immune system may not be the only culprit for salivary gland dysfunction. Some hypofunctional salivary glands from SS patients display acinar cell atrophy, loss of E-cadherin junctions, mislocalization of proteins, dilated ducts and remodeling of the ECM, and the extent of such pathology, including the degree of secretory defects, does not always correspond to the level of lymphocytic infiltrations. ${ }^{37-39}$ Therefore, at least in some patients, defective secretion and autoimmunity are not linked. ${ }^{1,40}$

During SMG embryonic development, we observed the Hippo pathway effector, TAZ, to be localized at cell-cell junctions in the polarized acinar progenitor cell layer. Moreover, in the newly forming secondary duct regions, a fraction of TAZ changed distribution from diffuse in the cytoplasm to a more focused organization at intercellular junctions where it colocalized with F-actin. This junctional localization of TAZ is supported by the observed increases in TAZ phosphorylation during embryonic branching morphogenesis. Moreover, colocalization of TAZ with F-actin in duct-forming regions coincided with a progressive increase in the interaction of TAZ with E-cadherin and $\alpha$-catenin. Taken together, these observations suggest a role for junctional TAZ in defining polarity in secondary duct cells. Consistent with this idea, knockdown of Lats2 altered the localization of TAZ and resulted in defects in branching morphogenesis and in the formation of secondary ductal structures. Moreover, NOD embryonic SMGs displayed similar features as the Lats2 siRNA-treated glands, providing evidence that deregulation of the Hippo pathway may contribute to structural defects in the NOD SMG. We also note that at E15.5, a fraction of TAZ was nuclear, supporting its role as a transcriptional coactivator of genes involved in remodeling the ECM at this stage of SMG cytodifferentiation. ${ }^{41}$ Further, a pool of TAZ was localized to the basal layer and the surrounding mesenchymal/ myoepithelial regions, suggesting an unexplored non- junctional and non-nuclear role for TAZ during SMG development. Our findings resemble studies demonstrating the importance of TAZ in normal alveolarization of lungs in mice, another tissue that develops through branching morphogenesis. ${ }^{42}$

E-cadherin has a pivotal role in the establishment of cell polarity in epithelial tissues. Our previous studies have documented that cytodifferentiation of the SMG is accompanied by increased interactions between $\alpha$-catenin and E-cadherin. ${ }^{16}$ Cellular localization of YAP, a paralog of TAZ, is regulated by binding to E-cadherin and $\alpha$-catenin. ${ }^{22-24}$ Here, we demonstrate that similar to YAP, TAZ is associated with $\alpha$-catenin and E-cadherin complexes, suggesting similar regulation of its subcellular localization. Interactions between TAZ and E-cadherin complexes in the SMG are likely to be mediated by $\alpha$-catenin, although it is also possible that a fraction of $\alpha$-catenin associates with TAZ independently of E-cadherin. The increase in the interactions of TAZ with E-cadherin and $\alpha$-catenin with SMG ctyodifferentiation strongly suggests that the Hippo pathway is important for maintaining junctional integrity and polarity during SMG-branching morphogenesis. Importantly, human labial SS tissues exhibit structural defects in the acini and ducts with the loss of E-cadherin and its associated junctional partners. This is consistent with the reduced E-cadherin and altered cell polarity reported in other SS studies. ${ }^{37,43-45}$ Defects in cell polarity in SS may have an impact on Hippo signaling that will ultimately affect downstream transcriptional events. Alternatively, inhibition of Hippo signaling may drive aberrant localization of TAZ to the nucleus and alter transcription, leading to ECM remodeling. As TAZ functions as a transcriptional coactivator of CTGF, a key factor contributing to tissue fibrosis, regulation of its subcellular localization is likely to have an important role in SS. Indeed, in the mature SMG, cells in the intercalated duct regions immediately adjacent to the acini are thought to function in acinar cell replacement during tissue renewal. Thus, defects in ductal cell polarity may have a role later in adult tissue homeostasis and be exacerbated by hormonal conditions and/or environmental insults, potentially explaining the frequently observed late onset of this disease.

When examining patients with different degrees of inflammation, we found tissue integrity among the biopsies to be largely heterogeneous, with structural defects evident in regions of the glands not adjacent to infiltrates. Although loss of structural integrity has been linked to hyposecretion in some SS cases, its role in increased susceptibility to lymphocytic infiltrations has not been explored. Interestingly, ablation of E-cadherin junctional protein, $\alpha$-catenin, has been shown to drive lymphocytic infiltration both in a mouse model and in a variety of cancers. ${ }^{4-48}$ Further studies are needed to determine how structural defects and loss of polarity in the SMG may lead to increased susceptibility to autoimmune reaction. 
To date, the etiology of SS remains controversial, and a common etiopathogenic model involves genetic predispositions triggered by environmental, pathological, and immunological factors that drive clinical manifestations, where loss of secretory function is believed to result from cytotoxicity caused by progressive lymphocytic infiltration. ${ }^{49}$ Here, we provide a molecular explanation for at least a subset of SS cases by demonstrating that the dysregulation of the Hippo pathway and associated tissue structural integrity may be an underlying basis of this disease.

\section{ACKNOWLEDGMENTS}

Grant sponsor: NIH; RO1 Grant number DE 014437 (MAK and ASM). Sjogren's Syndrome Foundation Research Grant (MAK), and March of Dimes Foundation; Research Grant number 5-FY11-578 (XV).

\section{DISCLOSURE/CONFLICT OF INTEREST}

The authors declare no conflict of interest.

1. Jonsson R, Bolstad Al, Brokstad KA, et al. Sjogren's syndrome-a plethora of clinical and immunological phenotypes with a complex genetic background. Ann N Y Acad Sci 2007;1108:433-447.

2. Hatzopoulos S, Amoroso C, Aimoni C, et al. Hearing loss evaluation of Sjogren's syndrome using distortion product otoacoustic emissions. Acta Otolaryngol Suppl 2002;548:20-25.

3. Boki KA, loannidis JP, Segas JV, et al. How significant is sensorineural hearing loss in primary Sjogren's syndrome? An individually matched case-control study. J Rheumatol 2001;28:798-801.

4. Ferrer JP, Biurrun $\mathrm{O}$, Lorente $\mathrm{J}$, et al. Auditory function in young patients with type 1 diabetes mellitus. Diabetes Res Clin Pract 1991; 11:17-22.

5. Fukushima $\mathrm{H}$, Cureoglu S, Schachern PA, et al. Cochlear changes in patients with type 1 diabetes mellitus. Otolaryngol Head Neck Surg 2005;133:100-106.

6. Beppu T, Sasaki T, Kawabata K, et al. Usefulness and limitations in ultrasonography for diagnosing neck lymph node metastases in patients with hypopharyngeal squamous cell carcinoma: comparison with pathological findings following neck dissection. Nihon Jibiinkoka Gakkai Kaiho 2005:108:794-800.

7. Lonyai A, Kodama S, Burger D, et al. The promise of Hox11+ stem cells of the spleen for treating autoimmune diseases. Horm Metab Res 2008;40:137-146.

8. Lonyai $A$, Kodama $S$, Burger $D$, et al. Fetal Hox11 expression patterns predict defective target organs: a novel link between developmental biology and autoimmunity. Immunol Cell Biol 2008;86:301-309.

9. Jaskoll T, Zhou YM, Chai Y, et al. Embryonic submandibular gland morphogenesis: stage-specific protein localization of FGFs, BMPs, Pax 6 and Pax9 in normal mice and abnormal SMG phenotypes in FgfR2IIIC(+/Delta), BMP7 $(-/-)$ and Pax6 $(-/-)$ mice. Cells Tissues Organs 2002;170:83-98.

10. Haara O, Koivisto T, Miettinen PJ. EGF-receptor regulates salivary gland branching morphogenesis by supporting proliferation and maturation of epithelial cells and survival of mesenchymal cells. Differentiation 2009:77:298-306.

11. Entesarian $\mathrm{M}$, Matsson $\mathrm{H}$, Klar J, et al. Mutations in the gene encoding fibroblast growth factor 10 are associated with aplasia of lacrimal and salivary glands. Nat Genet 2005;37:125-127.

12. Rebustini IT, Patel VN, Stewart JS, et al. Laminin alpha5 is necessary for submandibular gland epithelial morphogenesis and influences FGFR expression through beta1 integrin signaling. Dev Biol 2007;308:15-29.

13. Daley WP, Gervais EM, Centanni SW, et al. ROCK1-directed basement membrane positioning coordinates epithelial tissue polarity. Development 2012;139:411-422.

14. Knox SM, Lombaert IM, Reed X, et al. Parasympathetic innervation maintains epithelial progenitor cells during salivary organogenesis. Science 2010;329:1645-1647.

15. Patel VN, Rebustini IT, Hoffman MP. Salivary gland branching morphogenesis. Differentiation 2006;74:349-364.
16. Walker JL, Menko AS, Khalil S, et al. Diverse roles of E-cadherin in the morphogenesis of the submandibular gland: insights into the formation of acinar and ductal structures. Dev Dyn 2008;237: 3128-3141.

17. Cherrett C, Furutani-Seiki M, Bagby S. The Hippo pathway: key interaction and catalytic domains in organ growth control, stem cell self-renewal and tissue regeneration. Essays Biochem 2012;53: 111-127.

18. Varelas X, Wrana JL. Coordinating developmental signaling: novel roles for the Hippo pathway. Trends Cell Biol 2012;22:88-96.

19. Zhao B, Lei QY, Guan KL. The Hippo-YAP pathway: new connections between regulation of organ size and cancer. Curr Opin Cell Biol 2008;20:638-646.

20. Halder G, Johnson RL. Hippo signaling: growth control and beyond. Development 2011;138:9-22.

21. Lei QY, Zhang $\mathrm{H}$, Zhao $\mathrm{B}$, et al. TAZ promotes cell proliferation and epithelial-mesenchymal transition and is inhibited by the hippo pathway. Mol Cell Biol 2008;28:2426-2436.

22. Silvis MR, Kreger BT, Lien $\mathbf{W H}$, et al. Alpha-catenin is a tumor suppressor that controls cell accumulation by regulating the localization and activity of the transcriptional coactivator Yap1. Sci Signal 2011;4, ra33.

23. Kim NG, Koh E, Chen X, et al. E-cadherin mediates contact inhibition of proliferation through Hippo signaling-pathway components. Proc Natl Acad Sci USA. 2011;108:11930-11935.

24. Schlegelmilch K, Mohseni M, Kirak O, et al. Yap1 acts downstream of alpha-catenin to control epidermal proliferation. Cell 2011;144: 782-795

25. Varelas $X$, Samavarchi-Tehrani $P$, Narimatsu $M$, et al. The Crumbs complex couples cell density sensing to Hippo-dependent control of the TGF-beta-SMAD pathway. Dev Cell 2010;19: 831-844

26. Menko AS, Zhang L, Schiano F, et al. Regulation of cadherin junctions during mouse submandibular gland development. Dev Dyn 2002;224: 321-333.

27. Cutler LS, Mooradian BA. Lumen formation during development of the rat submandibular gland. J Dent Res 1987;66:1559-1562.

28. Heida Y, Nakanishi Y. Epithelial morphogenesis in mouse embryonic submandibular gland: its relationships to the tissue organization of epithelium and mesenchyme. Dev Growth Differ 1997; 39:1-8.

29. Remue $\mathrm{E}$, Meerschaert $\mathrm{K}$, Oka $\mathrm{T}$, et al. TAZ interacts with zonula occludens- 1 and -2 proteins in a PDZ-1 dependent manner. FEBS Lett 2010;584:4175-4180.

30. Oka $\mathrm{T}$, Remue $\mathrm{E}$, Meerschaert $\mathrm{K}$, et al. Functional complexes between YAP2 and ZO-2 are PDZ domain-dependent, and regulate YAP2 nuclear localization and signalling. Biochem J 2010;432: 461-472.

31. Soyfoo MS, Steinfeld S, Delporte C. Usefulness of mouse models to study the pathogenesis of Sjogren's syndrome. Oral Dis 2007;13: 366-375.

32. Kuroda S, Fukata M, Nakagawa M, et al. Role of IQGAP1, a target of the small GTPases Cdc42 and Rac1, in regulation of E-cadherin- mediated cell-cell adhesion. Science 1998;281:832-835.

33. Abdel-Wahab N, Weston BS, Roberts T, et al. Connective tissue growth factor and regulation of the mesangial cell cycle: role in cellular hypertrophy. J Am Soc Nephrol 2002;13:2437-2445.

34. Kodama $\mathrm{S}$, Kuhtreiber W, Fujimura $\mathrm{S}$, et al. Islet regeneration during the reversal of autoimmune diabetes in NOD mice. Science 2003;302:1223-1227.

35. Cha S, van Blockland SC, Versnel MA, et al. Abnormal organogenesis in salivary gland development may initiate adult onset of autoimmune exocrinopathy. Exp Clin Immunogenet 2001;18:143-160.

36. Price EJ, Venables PJ. The etiopathogenesis of Sjogren's syndrome. Arthritis Rheum 1995;25:117-133.

37. Ewert $\mathrm{P}$, Aguilera $\mathrm{S}$, Alliende $\mathrm{C}$, et al. Disruption of tight junction structure in salivary glands from Sjogren's syndrome patients is linked to proinflammatory cytokine exposure. Arthritis Rheum 2010; 62:1280-1289.

38. Velozo J, Aguilera S, Alliende C, et al. Severe alterations in expression and localisation of \{alpha\}6\{beta\}4 integrin in salivary gland acini from patients with Sjogren syndrome. Ann Rheum Dis 2009;68:991-996. 
39. Barrera MJ, Sanchez M, Aguilera S, et al. Aberrant localization of fusion receptors involved in regulated exocytosis in salivary glands of Sjogren's syndrome patients is linked to ectopic mucin secretion. J Autoimmun 2012;39:83-92.

40. Delaleu N, Jonsson MV, Appel S, et al. New concepts in the pathogenesis of Sjogren's syndrome. Rheum Dis Clin North Am 2008;34:833-845, vii.

41. Hoffman MP, Kidder BL, Steinberg ZL, et al. Gene expression profiles of mouse submandibular gland development: FGFR1 regulates branching morphogenesis in vitro through BMP- and FGF-dependent mechanisms. Development 2002;129:5767-5778.

42. Mitani A, Nagase T, Fukuchi K, et al. Transcriptional coactivator with PDZ-binding motif is essential for normal alveolarization in mice. Am J Respir Crit Care Med 2009;180:326-338.

43. Steinfeld S, Cogan E, King LS, et al. Abnormal distribution of aquaporin-5 water channel protein in salivary glands from Sjogren's syndrome patients. Lab Invest 2001;81:143-148.
44. Perez $\mathrm{P}$, Aguilera $\mathrm{S}$, Olea $\mathrm{N}$, et al. Aberrant localization of ezrin correlates with salivary acini disorganization in Sjogren's Syndrome. Rheumatology 2010;49:915-923.

45. Bahamondes V, Albornoz A, Aguilera S, et al. Changes in Rab3D expression and distribution in the acini of Sjogren's syndrome patients are associated with loss of cell polarity and secretory dysfunction. Arthritis Rheum 2011;63:3126-3135.

46. Tinkle $\mathrm{CL}$, Lechler $\mathrm{T}$, Pasolli $\mathrm{HA}$, et al. Conditional targeting of E-cadherin in skin: Insights into hyperproliferative and degenerative responses. Proc Natl Acad Sci 2003;101:552-557.

47. Vasioukhin VBC, Degenstein L, Wise B, et al. Hyperproliferation and defects in epithelial polarity upon conditional ablation of alphacatenin in skin. Cell 2001;104:605-617.

48. Balkwill $F$, Mantovani A. Inflammation and cancer: back to Virchow? Lancet 2001;357:539-545.

49. Price EJ, Venables PJ. The etiopathogenesis of Sjogren's syndrome. Semin Arthritis Rheum 1995;25:117-133. 